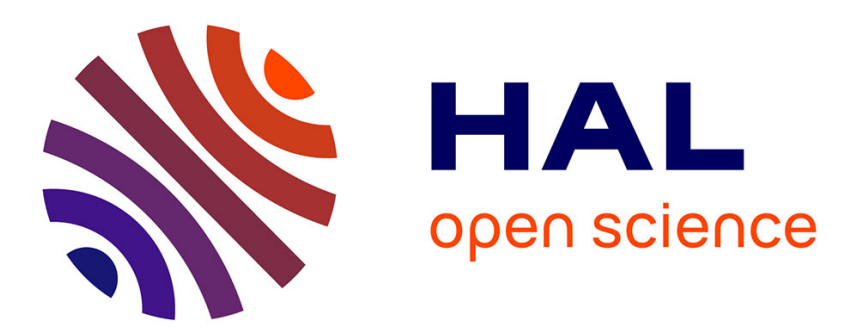

\title{
Superrotation of Venus' atmosphere analysed with a full general circulation model
}

Sébastien Lebonnois, Frédéric Hourdin, Vincent Eymet, Audrey Crespin, Richard Fournier, François Forget

\section{- To cite this version:}

Sébastien Lebonnois, Frédéric Hourdin, Vincent Eymet, Audrey Crespin, Richard Fournier, et al.. Superrotation of Venus' atmosphere analysed with a full general circulation model. Journal of Geophysical Research. Planets, 2010, 115 (E16), pp.E06006. 10.1029/2009JE003458 . hal-00535673

\section{HAL Id: hal-00535673 \\ https://hal.science/hal-00535673}

Submitted on 4 Jan 2022

HAL is a multi-disciplinary open access archive for the deposit and dissemination of scientific research documents, whether they are published or not. The documents may come from teaching and research institutions in France or abroad, or from public or private research centers.
L'archive ouverte pluridisciplinaire HAL, est destinée au dépôt et à la diffusion de documents scientifiques de niveau recherche, publiés ou non, émanant des établissements d'enseignement et de recherche français ou étrangers, des laboratoires publics ou privés.

$$
\text { Copyright }
$$




\title{
Superrotation of Venus' atmosphere analyzed with a full general circulation model
}

\author{
Sébastien Lebonnois, ${ }^{1}$ Frédéric Hourdin, ${ }^{1}$ Vincent Eymet, ${ }^{2}$ Audrey Crespin, ${ }^{1}$ \\ Richard Fournier, ${ }^{2}$ and François Forget ${ }^{1}$ \\ Received 26 June 2009; revised 16 October 2009; accepted 13 January 2010; published 12 June 2010.
}

[1] A general circulation model (GCM) has been developed for the Venus atmosphere, from the surface up to $100 \mathrm{~km}$ altitude, based on the GCM developed for Earth at our laboratory. Key features of this new GCM include topography, diurnal cycle, dependence of the specific heat on temperature, and a consistent radiative transfer module based on net exchange rate matrices. This allows a consistent computation of the temperature field, in contrast to previous GCMs of Venus atmosphere that used simplified temperature forcing. The circulation is analyzed after 350 Venus days (111 Earth years). Superrotation is obtained above roughly $40 \mathrm{~km}$ altitude. Below, the zonal wind remains very small compared to observed values, which is a major pending question. The meridional circulation consists of equator-to-pole cells, the dominant one being located within the cloud layers. The modeled temperature structure is globally consistent with observations, though discrepancies persist in the stability of the lowest layers and equator-pole temperature contrast within the clouds ( $10 \mathrm{~K}$ in the model compared to the observed $40 \mathrm{~K}$ ). In agreement with observational data, a convective layer is found between the base of the clouds (around $47 \mathrm{~km}$ ) and the middle of the clouds (55-60 km altitude). The transport of angular momentum is analyzed, and comparison between the reference simulation and a simulation without diurnal cycle illustrates the role played by thermal tides in the equatorial region. Without diurnal cycle, the Gierasch-Rossow-Williams mechanism controls angular momentum transport. The diurnal tides add a significant downward transport of momentum in the equatorial region, causing low latitude momentum accumulation.

Citation: Lebonnois, S., F. Hourdin, V. Eymet, A. Crespin, R. Fournier, and F. Forget (2010), Superrotation of Venus' atmosphere analyzed with a full general circulation model, J. Geophys. Res., 115, E06006, doi:10.1029/2009JE003458.

\section{Introduction}

[2] With the successful Venus Express European mission to Venus, and the perspective of other upcoming missions to Venus, such as the Japanese Akatsuki mission (or Venus Climate Orbiter), a renewed interest in the Venusian climate has appeared in the last few years, especially with the development of several general circulation models around the world. The atmosphere of Venus exhibits several peculiar characteristics that have been revealed by the series of spacecrafts and probes sent for exploration during the 1970s and 1980s (Venera missions series, Pioneer Venus Orbiter and probes, Magellan). Predominant features are the dense atmosphere, with 92 bars of $\mathrm{CO}_{2}(96.5 \%)$ at the surface; the dense cloud layers, which cover the whole

\footnotetext{
${ }^{1}$ Laboratoire de Météorologie Dynamique, UMR 8539, IPSL, UPMC, CNRS, Paris, France.

${ }^{2}$ Laboratoire Plasma et Conversion de l'Energie, UMR 5213, UPS, CNRS, Toulouse, France.

Copyright 2010 by the American Geophysical Union. 0148-0227/10/2009JE003458
}

planet in the altitude range 48 to $70 \mathrm{~km}$; the extremely hot deep atmosphere, with roughly $735 \mathrm{~K}$ at the surface; the fast rotation rate of the whole atmosphere, which spins in the same direction as the solid surface with zonal winds peaking at more than $100 \mathrm{~m} \mathrm{~s}^{-1}$ near the cloud top (superrotation) and the vortex structures centered around both poles above $60^{\circ}$ latitude. The most recent comprehensive reviews of the thermal structure and dynamics of Venus' atmosphere may be found in the work by Crisp and Titov [1997], Zasova et al. [2007] and Gierasch et al. [1997].

[3] The mechanisms that may explain the maintenance of such a superrotation have been explored through many model developments, starting thirty years ago with the pioneering three-dimensional general circulation model by Young and Pollack [1977]. This model was able to get substantial superrotation above $30 \mathrm{~km}$ altitude, despite its low resolution and potential problems in its vertical diffusion formulation [Rossow et al., 1980; Young and Pollack, 1980]. Theoretical studies have put forward two mechanisms that may help interpret the superrotation. The first one is called the Gierasch-Rossow-Williams mechanism (GRW) [from Gierasch, 1975; Rossow and Williams, 1979]: the 
mean meridional circulation, which consists of thermally direct cells with ascending motions around equator and descending flows in polar regions, transports angular momentum upward, and toward high latitudes, inducing high zonal speed jets that become unstable. Waves, possibly generated through barotropic instabilities along the equatorward side of the jet, redistribute angular momentum equatorward. The second hypothesis involves the diurnal motion of the Sun, which may affect the angular momentum budget through two different mechanisms. A first possibility is the moving flame mechanism [Schubert and Whitehead, 1969; Schubert, 1983] where tilted eddies induced by the rotating heat from the Sun transport angular momentum upward. A second possibility is the thermal tides generated within and above the cloud layers, which have been shown to be able to play a major part in the maintenance of the superrotation [Pechman and Ingersoll, 1984; Fels, 1986; Leovy and Baker, 1987; Hou et al., 1990; Newman and Leovy, 1992; Takagi and Matsuda, 2007]. The respective roles of each of these mechanisms have been investigated using general circulation models (GCMs). Del Genio et al. [1993] and Del Genio and Zhou [1996] used an Earth-like GCM with the rotation period of Venus, which could produce superrotation through the GRW mechanism when upper and lower atmospheres were strongly decoupled. The GCM developed for Titan (Saturn's largest satellite, which has also a superrotating atmosphere) at the Laboratoire de Météorologie Dynamique [Hourdin et al., 1992, 1995, 2004; Rannou et al., 2002, 2004] has also been able to produce strong superrotation in this atmosphere, through the GRW mechanism. However, the atmosphere of Venus had never been fully simulated, with all its properties and characteristics.

[4] Recently, Venus GCMs have been developed worldwide, using simplified physical and radiative parameterizations. The models developed by Yamamoto and Takahashi [2003, 2004, 2006] and Hollingsworth et al. [2007] force atmospheric heating rates and relax the temperature toward a specified temperature profile (Newtonian cooling). In these simulations, surface-to-cloud-top and equator-to-pole Hadley circulation is generated, and significant superrotation is obtained, though the thermal forcing needs to be stronger than expected, with heating rates in the lower atmosphere of the order of $0.5 \mathrm{~K} /$ Earth day, compared to values of the order of $10^{-3} \mathrm{~K} /$ Earth day suggested by observations [Tomasko et al., 1980]. The model developed in Oxford [Lee, 2006; Lee et al., 2005, 2007], as well as the model developed by Herrnstein and Dowling [2007], use a relaxation of the temperature toward a specified temperature structure that includes latitudinal variations. The equator-to-pole temperature gradient forced in this structure for the lower atmosphere is of the order of $10 \mathrm{~K}$. As in the other models, significant superrotation is obtained, with similar Hadley circulation.

[5] The self-consistent computation of temperature using a true radiative transfer code (and thus a realistic radiative forcing) is a much more difficult task, and only two models have been developed so far: Ikeda et al. [2007] and the one used in this paper [Lebonnois et al., 2005]. Here, we present the first results of this new GCM developed for Venus, which includes a full radiative transfer module, allowing the temperature structure to be computed self-consistently. This radiative transfer model [Eymet et al., 2009] represents a significant step forward in the simulation of a realistic Venus' atmosphere.

[6] In section 2, the general circulation model is detailed, including a summary of the radiative transfer module's characteristics. Results concerning the mean meridional circulation are developed in section 3, where we insist on the role of realistic radiative transfer. Angular momentum transport and superrotation mechanisms are then analyzed in section 4 .

\section{General Circulation Model Description}

\subsection{Dynamical Core and Parameterizations}

[7] The dynamical core of our general circulation model is based on the LMDZ Earth model developed at the Laboratoire de Météorologie Dynamique [Hourdin et al., 2006]. The $Z$ stands for "zoom" because this model has the ability to zoom the grid over a given region if required. The finite difference discretization scheme conserves both potential enstrophy for barotropic nondivergent flows, and total angular momentum for axisymmetric flow. For latitudes poleward of $60^{\circ}$ in both hemisphere, a longitudinal filter is applied to limit the effective resolution to that at $60^{\circ}$. In the current version of the Venus GCM, we use a horizontal resolution of 48 longitudes $\times 32$ latitudes $\left(7.5^{\circ} \times\right.$ $\left.5.625^{\circ}\right)$. The time step is 1.5 minutes, and the model uses a Leapfrog time integration scheme, with a periodic predictorcorrector time step.

[8] The vertical grid is based on hybrid coordinates, with pressure at level $j$ defined as $p(j)=a p(j)+b p(j) \times p_{\text {surf }}$. The coefficients $a p$ and $b p$ are given in Table 1 for the 50 levels of the GCM, together with the corresponding pressure for a surface pressure of 92 bar, and the corresponding altitude, first following the Venus International Reference Atmosphere (VIRA) [Seiff et al., 1985] and second computed from the globally averaged geopotential in the reference simulation of the GCM (this simulation is detailed in section 2.3).

[9] The orbit of Venus is taken as circular, and we neglect the inclination of Venus' rotation axis. Model parameters are collected in Table 2.

[10] The standard version of the LMDZ dynamical core uses a single value for the specific heat $C_{p}$, but $C_{p}$ varies in the atmosphere of Venus from $738 \mathrm{~J} / \mathrm{kg} / \mathrm{K}$ at $100 \mathrm{~km}$ altitude to $1181 \mathrm{~J} / \mathrm{kg} / \mathrm{K}$ near the surface (values taken from the Venus International Reference Atmosphere [Seiff et al., 1985]). This variation of $C_{p}$ with temperature needs to be taken into account, in order to get realistic adiabatic lapse rates in the whole atmosphere. We use an analytic approximation for this temperature dependence, that yields values very close to the VIRA profile for $C_{p}$ (within $4 \%$ for temperatures below $200 \mathrm{~K}$, below $1 \%$ everywhere else):

$$
C_{p}(T)=C_{p_{0}} \times\left(\frac{T}{T_{0}}\right)^{\nu},
$$

with $C_{p 0}=1000 \mathrm{~J} / \mathrm{kg} / \mathrm{K}, T_{0}=460 \mathrm{~K}$, and $\nu=0.35$.

[11] In the LMDZ model, the potential temperature is one of the fundamental prognostic variable in the equations of 
Table 1. Hybrid Coefficients for the 50 Vertical Levels of the GCM and Corresponding Pressure and Altitude ${ }^{\mathrm{a}}$

\begin{tabular}{|c|c|c|c|c|c|}
\hline \multirow[b]{2}{*}{ Level } & \multirow[b]{2}{*}{$a p(\mathrm{~Pa})$} & \multirow[b]{2}{*}{$b p$} & \multirow[b]{2}{*}{ Pressure $(\mathrm{hPa})$} & \multicolumn{2}{|r|}{ Altitude $(\mathrm{km})$} \\
\hline & & & & VIRA $^{b}$ & Reference GCM Simulation $^{c}$ \\
\hline 1 & 0. & 1. & $9.200 \times 10^{4}$ & 0.00 & 0.00 \\
\hline 2 & 656. & $9.99 \times 10^{-1}$ & $9.188 \times 10^{4}$ & 0.04 & 0.03 \\
\hline 3 & $2.61 \times 10^{+3}$ & $9.95 \times 10^{-1}$ & $9.155 \times 10^{4}$ & 0.10 & 0.12 \\
\hline 4 & $7.67 \times 10^{+3}$ & $9.85 \times 10^{-1}$ & $9.067 \times 10^{4}$ & 0.25 & 0.32 \\
\hline 5 & $1.73 \times 10^{+4}$ & $9.66 \times 10^{-1}$ & $8.901 \times 10^{4}$ & 0.54 & 0.68 \\
\hline 6 & $3.30 \times 10^{+4}$ & $9.35 \times 10^{-1}$ & $8.636 \times 10^{4}$ & 1.02 & 1.23 \\
\hline 7 & $5.57 \times 10^{+4}$ & $8.91 \times 10^{-1}$ & $8.256 \times 10^{4}$ & 1.72 & 2.03 \\
\hline 8 & $8.63 \times 10^{+4}$ & $8.33 \times 10^{-1}$ & $7.754 \times 10^{4}$ & 2.69 & 3.10 \\
\hline 9 & $1.25 \times 10^{+5}$ & $7.62 \times 10^{-1}$ & $7.135 \times 10^{4}$ & 3.97 & 4.50 \\
\hline 10 & $1.70 \times 10^{+5}$ & $6.79 \times 10^{-1}$ & $6.413 \times 10^{4}$ & 5.58 & 6.23 \\
\hline 11 & $2.21 \times 10^{+5}$ & $5.86 \times 10^{-1}$ & $5.613 \times 10^{4}$ & 7.55 & 8.35 \\
\hline 12 & $2.75 \times 10^{+5}$ & $4.89 \times 10^{-1}$ & $4.772 \times 10^{4}$ & 9.90 & 10.8 \\
\hline 13 & $3.27 \times 10^{+5}$ & $3.91 \times 10^{-1}$ & $3.928 \times 10^{4}$ & 12.6 & 13.7 \\
\hline 14 & $3.74 \times 10^{+5}$ & $2.99 \times 10^{-1}$ & $3.122 \times 10^{4}$ & 15.8 & 17.0 \\
\hline 15 & $4.13 \times 10^{+5}$ & $2.15 \times 10^{-1}$ & $2.389 \times 10^{4}$ & 19.2 & 20.7 \\
\hline 16 & $4.40 \times 10^{+5}$ & $1.43 \times 10^{-1}$ & $1.754 \times 10^{4}$ & 23.1 & 24.6 \\
\hline 17 & $4.52 \times 10^{+5}$ & $8.75 \times 10^{-2}$ & $1.257 \times 10^{4}$ & 27.0 & 28.3 \\
\hline 18 & $4.50 \times 10^{+5}$ & $4.90 \times 10^{-2}$ & $9.008 \times 10^{3}$ & 30.7 & 31.9 \\
\hline 19 & $4.35 \times 10^{+5}$ & $2.29 \times 10^{-2}$ & $6.454 \times 10^{3}$ & 34.1 & 35.2 \\
\hline 20 & $4.01 \times 10^{+5}$ & $6.66 \times 10^{-3}$ & $4.635 \times 10^{3}$ & 37.4 & 38.4 \\
\hline 21 & $3.29 \times 10^{+5}$ & $2.67 \times 10^{-4}$ & $3.314 \times 10^{3}$ & 40.5 & 41.4 \\
\hline 22 & $2.37 \times 10^{+5}$ & $5.38 \times 10^{-8}$ & $2.374 \times 10^{3}$ & 43.4 & 44.2 \\
\hline 23 & $1.70 \times 10^{+5}$ & $2.69 \times 10^{-15}$ & $1.701 \times 10^{3}$ & 46.3 & 46.9 \\
\hline 24 & $1.22 \times 10^{+5}$ & 0 & $1.219 \times 10^{3}$ & 49.0 & 49.5 \\
\hline 25 & $8.73 \times 10^{+4}$ & 0. & $8.735 \times 10^{2}$ & 51.5 & 51.9 \\
\hline 26 & $6.26 \times 10^{+4}$ & 0. & $6.259 \times 10^{2}$ & 53.9 & 54.1 \\
\hline 27 & $4.48 \times 10^{+4}$ & 0. & $4.485 \times 10^{2}$ & 56.1 & 56.2 \\
\hline 28 & $3.21 \times 10^{+4}$ & 0. & $3.213 \times 10^{2}$ & 58.2 & 58.1 \\
\hline 29 & $2.30 \times 10^{+4}$ & 0. & $2.302 \times 10^{2}$ & 60.1 & 60.1 \\
\hline 30 & $1.65 \times 10^{+4}$ & 0. & $1.650 \times 10^{2}$ & 62.0 & 61.9 \\
\hline 31 & $1.18 \times 10^{+4}$ & 0. & $1.182 \times 10^{2}$ & 63.9 & 63.7 \\
\hline 32 & $8.47 \times 10^{+3}$ & 0. & 84.70 & 65.7 & 65.5 \\
\hline 33 & $6.07 \times 10^{+3}$ & 0. & 60.69 & 67.4 & 67.2 \\
\hline 34 & $4.35 \times 10^{+3}$ & 0. & 43.49 & 69.2 & 68.8 \\
\hline 35 & $3.12 \times 10^{+3}$ & 0. & 31.16 & 70.8 & 70.5 \\
\hline 36 & $2.23 \times 10^{+3}$ & 0. & 22.33 & 72.5 & 72.2 \\
\hline 37 & $1.60 \times 10^{+3}$ & 0. & 16.00 & 74.1 & 73.8 \\
\hline 38 & $1.15 \times 10^{+3}$ & 0. & 11.46 & 75.7 & 75.5 \\
\hline 39 & $8.21 \times 10^{+2}$ & 0. & 8.214 & 77.3 & 77.1 \\
\hline 40 & $5.89 \times 10^{+2}$ & 0. & 5.886 & 78.8 & 78.7 \\
\hline 41 & $4.22 \times 10^{+2}$ & 0. & 4.217 & 80.3 & 80.2 \\
\hline 42 & $3.02 \times 10^{+2}$ & 0. & 3.022 & 81.7 & 81.8 \\
\hline 43 & $2.17 \times 10^{+2}$ & 0. & 2.165 & 83.1 & 83.3 \\
\hline 44 & $1.55 \times 10^{+2}$ & 0. & 1.551 & 84.4 & 84.8 \\
\hline 45 & $1.11 \times 10^{+2}$ & 0. & 1.112 & 85.8 & 86.2 \\
\hline 46 & 79.7 & 0. & 0.7965 & 87.1 & 87.8 \\
\hline 47 & 51.5 & 0. & 0.5150 & 88.8 & 90.1 \\
\hline 48 & 28.3 & 0. & 0.2826 & 91.0 & 92.9 \\
\hline 49 & 14.3 & 0. & 0.1429 & 93.3 & 94.9 \\
\hline 50 & 6.11 & 0. & 0.0611 & 95.5 & 101. \\
\hline
\end{tabular}

${ }^{a}$ Vertical levels are at the bottom of each layer. Corresponding pressure is for a surface pressure of 92 bar.

${ }^{\mathrm{b}}$ Calculated using the standard VIRA pressure/altitude model at equator [Seiff et al., 1985].

${ }^{\mathrm{c} C}$ Computed with the globally averaged geopotential from the reference GCM simulation.

energy conservation. The potential temperature $\theta$ is the temperature an air parcel initially at temperature $T$ and pressure $p$ would reach when brought to a reference pressure $p_{\text {ref }}$ (typically, the surface pressure) adiabatically:

$$
\int_{\theta}^{T} C_{p} \frac{d T}{T}=\int_{p_{\mathrm{ref}}}^{p} R \frac{d p}{p}
$$

( $R$ is the atmospheric gas constant). Equation (2) is obtained from the first principle of thermodynamics when a parcel of air at pressure $p$ and temperature $T$ is brought adiabatically to the reference pressure $p_{\text {ref. }}$.

[12] When $C_{p}$ is taken as constant with temperature, equation (2) yields the classical expression of potential temperature $\theta=T \times\left(p_{\text {ref }} / p\right)^{\kappa}$, with $\kappa=R / C_{p}$. However, when $C_{p}$ depends on temperature, this expression is no longer valid.

[13] To keep modifications of the dynamical core to the minimum, we have kept potential temperature as a key variable in the dynamics. Thus, we have calculated the new expression of potential temperature under these conditions. 
Table 2. List of the Main GCM Parameters

\begin{tabular}{|c|c|}
\hline Parameter & Value \\
\hline \multicolumn{2}{|c|}{ Venus Characteristics ${ }^{\mathrm{a}}$} \\
\hline Planet radius & $6051.3 \mathrm{~km}$ \\
\hline Gravity & $8.87 \mathrm{~m} \mathrm{~s}^{-2}$ \\
\hline Revolution period & 224.7 Earth days \\
\hline Rotation rate & $-2.99 \times 10^{-7} \mathrm{~s}$ \\
\hline Rotation period & -243 . Earth days \\
\hline Length of solar day & $1.0087 \times 10^{-7} \mathrm{~s}$ \\
\hline Obliquity (approximation) & $180^{\circ}$ \\
\hline Eccentricity (approximation) & 0 \\
\hline Distance to Sun & $108.15 \times 10^{6} \mathrm{~km}$ \\
\hline \multicolumn{2}{|c|}{ Atmospheric Properties ${ }^{\mathrm{a}}$} \\
\hline Mean surface pressure & $9.2 \times 10^{6} \mathrm{~Pa}$ \\
\hline Mean molecular mass & $43.44 \mathrm{~g} \mathrm{~mol}^{-1}$ \\
\hline \multicolumn{2}{|c|}{ Horizontal Dissipation Parameters } \\
\hline $\begin{array}{l}\text { Horizontal dissipation time } \\
\text { constant }\end{array}$ & $\begin{array}{r}2 \times 10^{4} \text { s below } \sim 3 \times 10^{6} \mathrm{~Pa} \\
1 \times 10^{4} \mathrm{~s} \text { above } \sim 3 \times 10^{6} \mathrm{~Pa}\end{array}$ \\
\hline $\begin{array}{l}\text { Number of iterations } \\
\text { (dissipative operator) }\end{array}$ & 2 \\
\hline \multicolumn{2}{|c|}{ Surface, Soil, and Boundary Layer Parameters } \\
\hline Surface albedo & 0.1 \\
\hline Surface thermal inertia & $2000 \mathrm{~J} \mathrm{~m}^{-2} \mathrm{~s}^{-0.5} \mathrm{~K}^{-1}$ \\
\hline Surface roughness coefficient & $0.01 \mathrm{~m}$ \\
\hline Mixing length & $35 \mathrm{~m}$ \\
\hline Minimum turbulent coefficient & 3. $\times 10^{-4} \mathrm{~m}^{2} \mathrm{~s}^{-1}$ \\
\hline
\end{tabular}

${ }^{\mathrm{a}}$ From, e.g., Schubert [1983].

Introducing the expression of $C_{p}(T)$ (equation (1)) in equation (2) yields

$$
\int_{\theta}^{T} \frac{T^{\nu-1}}{T_{0}^{\nu}} d T=\frac{R}{C_{p_{0}}} \ln \frac{p}{p_{\mathrm{ref}}}
$$

then (for $\nu \neq 0$ )

$$
\frac{1}{\nu T_{0}^{\nu}}\left(T^{\nu}-\theta^{\nu}\right)=\ln \left(\frac{p}{p_{\text {ref }}}\right)^{\kappa_{0}},
$$

where $\kappa_{0}=\frac{R}{C p_{0}}$. This yields the new expression for the potential temperature:

$$
\theta^{\nu}=T^{\nu}+\nu T_{0}^{\nu} \ln \left(\frac{p_{\text {ref }}}{p}\right)^{\kappa_{0}} .
$$

[14] The adjustments done in the dynamical core enable us to run the GCM with any formulation of $C_{p}$ and the corresponding potential temperature based on equation (2). In our reference simulation, equations (1) and (5) are taken into account. However, we made also simulations with a constant $C_{p}$ value, and the classical potential temperature (see section 2.3).

[15] When necessary, i.e., when the vertical temperature gradient is below the adiabatic lapse rate, dry convective adjustment occurs. Potential enthalpy $\left(H=C_{p} \times \theta\right)$ is mixed to keep the adiabatic lapse rate across the unstable layer. This convective adjustment is achieved through parcel exchange, and therefore these motions should also mix momentum. The intensity of this exchange of momentum cannot be evaluated from the simple model used here. It is thus estimated through a coefficient that depends on the amplitude of the enthalpy adjustment, so that the momentum exchange increases with the instability [Hourdin et al., 1993].

[16] The surface boundary layer parameterization used in this GCM is similar to the one used in the early threedimensional version of our Titan GCM [Hourdin et al., 1995], with parameters indicated in Table 2.

[17] The vertical diffusion flux of a quantity (by unit mass) $x$ is computed as $F=-\rho k(\partial x / \partial z)$, with $\rho$ the atmospheric density, and $k$ the diffusion coefficient. At the surface, the flux is $F=\rho V C_{d} \Delta x$, with $V$ the wind in the first layer of the model, and $C_{d}$ the surface drag coefficient, computed as

$$
C_{d}=\left(\frac{\kappa_{\mathrm{VK}}}{\ln \left(1+z_{1} / z_{0}\right)}\right)^{2} f\left(R_{i}\right),
$$

where $\kappa_{\mathrm{VK}}$ is the Von Karman constant $\left(\kappa_{\mathrm{VK}}=0.4\right), z_{1}$ is the altitude of the first layer in the model, $z_{0}$ is the rugosity or roughness length, a characteristic dimension of the roughness of the surface (chosen arbitrarily as $z_{0}=0.01 \mathrm{~m}$ ), and $f\left(R_{i}\right)$ is a function of instability depending on the Richardson number $R_{i}$. This function was defined for Earth [see Louis, 1979], and its validity here may be subject to caution.

[18] The diffusion coefficient $K_{z}$ is computed as

$$
K_{z}=h^{2}\left|\frac{\partial V}{\partial z}\right| \sqrt{\epsilon},
$$

where $h=l_{\text {mix }}\left(z_{2}-z\right) /\left(z_{2}-z_{1}\right), l_{\text {mix }}$ is a mixing length, chosen as $35 \mathrm{~m}, z_{2}$ is the altitude of the top layer of the model, $\epsilon=\left(R_{i}^{c}-R_{i}\right) / R_{i}^{c}, R_{i}^{c}$ is the critical Richardson number (chosen as 0.4 ). A minimum value is fixed for $K_{z}, K_{\min }=3 \times$ $10^{-4} \mathrm{~m}^{2} \mathrm{~s}^{-1}$ (stable conditions).

[19] The sensitivity of the simulations to this choice of parameterization, and of these parameters, need to be tested in depth. Several tests indicate that they indeed affect the circulation in the deep atmosphere. A full study is currently ongoing and will be reported in a future paper.

[20] For thermal conduction in the soil, we use a soil model (11 layers) [Hourdin et al., 1993]. The model depends on one parameter, the thermal inertia of the surface. The chosen thermal inertia $\left(I=2000 \mathrm{~J} \mathrm{~m}^{-2} \mathrm{~s}^{-0.5} \mathrm{~K}^{-1}\right)$ is typical for nonporous basalts [Zimbelman, 1986].

[21] A high-resolution topographical data set from the Magellan mission (PDS Magellan GTDR data set: http://pdsgeosciences.wustl.edu/missions/magellan/shadr_topo_grav/ index.htm [Ford and Pettengill, 1992]) was used to specify the surface topography smoothed to the GCM resolution. Evolved parameterizations have been developed for the Earth and Mars [Lott and Miller, 1997; Forget et al., 1999] to take into account subgrid topography effects (drag, lift and gravity waves), but these have not yet been included in the Venus GCM.

[22] Horizontal dissipation is taken into account, based on an iterated Laplacian. The dissipative time constants are chosen to minimize the impact of this dissipation while maintaining numerical stability of the simulation. The chosen values for the simulations presented in this work are $2 \times$ $10^{4} \mathrm{~s}$ below roughly $15 \mathrm{~km}$ altitude, and $1 \times 10^{4} \mathrm{~s}$ above, with a smoothed transition over a few layers. In the top four layers of the GCM, a sponge layer is included, with Rayleigh 
friction damping horizontal winds to zero. Time constants are $9.6 \times 10^{4} \mathrm{~s}(1.12$ Earth days $)$ for the top layer, then $1.20 \times 10^{5}$, $1.23 \times 10^{5}$, and $1.60 \times 10^{5} \mathrm{~s}$ for the three layers below, following values used by Newman and Leovy [1992].

\subsection{Radiative Transfer}

\subsubsection{Full Radiative Transfer Scheme}

[23] For solar radiation, heating rates are taken from lookup tables, with downward solar flux profiles computed as a function of solar zenith angle $\left(0^{\circ}\right.$ to $\left.95^{\circ}\right)$, based on Crisp [1986]. Interpolation is done for the correct solar zenith angle for each GCM grid point, taking into account the diurnal cycle.

[24] For thermal radiation, we used the code developed by Eymet et al. [2009] based on the net exchange rates (NER) matrix formalism [Eymet et al., 2005; Dufresne et al., 2005], in the wavelength range from 1.7 to $250 \mu \mathrm{m}$. The heating rate of a layer $i,-C_{i}$, is computed as the sum of net exchanges $\Psi(i, j, \lambda)$ with all the other layers $j$ (including space and ground) and in 68 wavelength narrow bins:

$$
-C_{i}=\sum_{\lambda} \sum_{j} \Psi(i, j, \lambda)
$$

The individual net exchange rates are computed with

$$
\Psi(i, j, \lambda)=\left(B\left(T_{i}, \lambda\right)-B\left(T_{j}, \lambda\right)\right) \xi(i, j, \lambda),
$$

where $B(T, \lambda)$ is the Planck function, and $\xi(i, j, \lambda)$ is an opticogeometric factor assumed to be temperature independent.

[25] Given atmospheric composition (assumed to be horizontally uniform), Eymet et al. [2009] compute correlated k coefficients for the gas. They also use a cloud model [Zasova et al., 2007] that prescribes particles properties and distributions (without taking into account any latitudinal variations). From these data, the NER coefficients $\xi(i, j, \lambda)$ are computed with a Monte Carlo code (see details in the work by Eymet et al. [2009]). This $\xi$ matrix is the input taken into account in the GCM, where the NER matrix $\Psi$ is computed at each radiative time step (2000 times per Venus day for the moment, i.e. every $1.4 \mathrm{~h}$ ), using the modeled temperature field and equation (9).

[26] As discussed by Eymet et al. [2009], the opacity in the deep atmosphere strongly affects the infrared net flux vertical profile, and therefore the temperatures obtained below the clouds. In order to get an average surface temperature close to the VIRA reference $(735 \mathrm{~K})$, we had to significantly increase the $\mathrm{CO}_{2}$ continuum opacity in the farinfrared (we multiplied this parameter by a factor of 6 over the thermal infrared region, not in the near-infrared windows where constraints exist). There is a clear need here to better constrain opacity sources in the deep atmosphere.

[27] To take into account the topography, $\xi$ matrices were computed for several surface pressures, from 40 to 115 bar (5 bar steps). For each GCM grid point, we interpolate the $\xi$ matrix linearly to the actual surface pressure at the beginning of the simulation.

[28] The cloud structure is taken as uniform for the moment, with a base at $47 \mathrm{~km}$ altitude, and a cloud top located around $70 \mathrm{~km}$ altitude. In the atmosphere of Venus, though, the clouds are variable. In particular, the cloud top has been observed to vary from roughly $60 \mathrm{~km}$ near the poles to $70 \mathrm{~km}$ equatorward of $50^{\circ}-60^{\circ}$ latitude [Zasova et al., 2007; Titov et al., 2008]. Taking into account a latitudinal variation of the cloud model would be quite easy to implement, as soon as characteristics of the clouds in the polar region are available. This would be a significant improvement to test the role of cloud variations in the dynamics of the polar regions.

\subsubsection{Simplified Radiative Forcing}

[29] To test our GCM under conditions similar to previously published models, we also performed simulations with a simplified radiative forcing. In this simplified scheme, the radiative tendency (temporal variation between two model time steps) on temperature at longitude $\lambda$, latitude $\phi$ and pressure level $p$ is given by

$$
\frac{\delta T_{\mathrm{rad}}(\lambda, \phi, p, t)}{\delta t}=-\frac{T(\lambda, \phi, p, t)-T_{0}(\phi, p)}{\tau},
$$

where $T_{0}(\phi, p)$ is the forcing thermal structure, and $\tau$ is the time constant of this forcing. $T_{0}(\phi, p)$ and $\tau$ are taken from Lee [2006]:

$$
T_{0}(\phi, p)=T_{\text {ref }}(p)+T_{1}(p)(\cos (\phi)-C),
$$

where $T_{\text {ref }}(p)$ is a reference temperature profile (VIRA model [Seiff et al., 1985]), and $T_{1}(p)$ is a perturbation term giving the peak equator-to-pole difference. The constant $C$ is the integral of $\cos (\phi)$ over the domain $(C=\pi / 4)$. The profile of $T_{1}(p)$ was chosen to reflect the peak in absorption of solar insolation within the cloud deck [Lee, 2006]. The value of $\tau$ is 25 Earth days, decreasing slightly in the uppermost levels. In this formulation, the diurnal cycle is not taken into account.

[30] For these simulations, simple lower boundary layer friction and vertical eddy diffusion coefficients are also implemented. In the lowest layer, Rayleigh friction relaxes the wind field toward zero with a time constant of 32 Earth days. The vertical eddy diffusion coefficient is fixed at $2.5 \times$ $10^{-2} \mathrm{~m}^{2} \mathrm{~s}^{-1}$.

\subsection{Simulations}

[31] A reference simulation was first run for 350 Venus days $\left(3.5 \times 10^{9} \mathrm{~s}\right.$, or 111 Earth years $)$, with topography and a full radiative transfer scheme including the diurnal cycle (heating rate tables depending on zenith angle, and $\xi$ matrices depending on surface pressure). The run was started with the atmosphere at rest (uniform zonal wind field $u=0$ ), with a vertical temperature profile very close to the VIRA model, uniform for every GCM grid point. Surface pressure (92 bar) and temperature profile are set for all grid points alike, despite the topography. The total mass of the atmosphere and initial total angular momentum are therefore the same in simulations both with and without topography. The pressure and temperature in the deepest layers adapt quickly to the topography. After 350 Venus days, the atmospheric state has converged toward a steady cycle, though the total angular momentum is still slightly increasing. The averaged net energy flux at the top of the atmosphere is around $1 \mathrm{~W} \mathrm{~m}^{-2}$.

[32] The atmospheric state after 250 Venus days is almost identical to the one obtained after 350 Venus days. We used that state as the starting point for sensitivity runs of 100 Venus days: (1) sensitivity to resolution with a $64 \times$ 


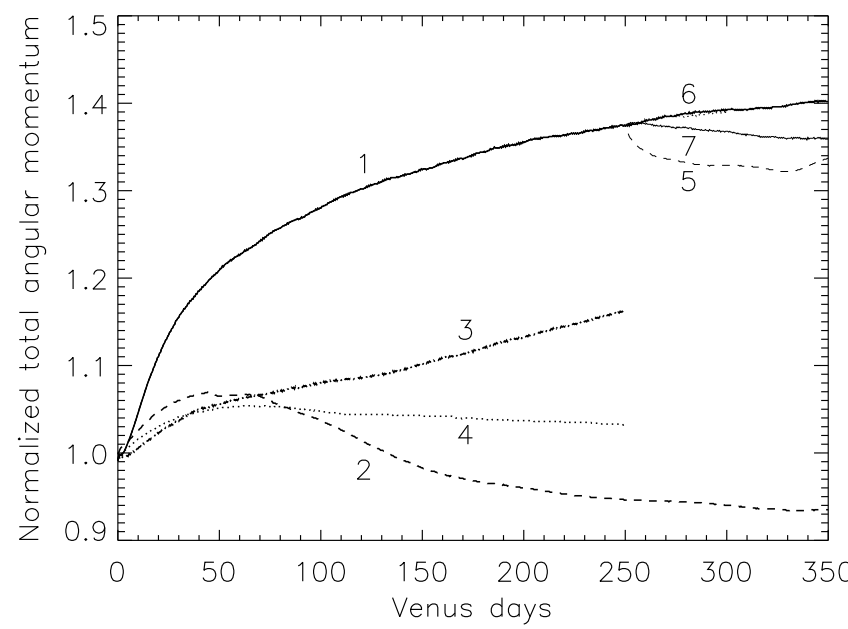

Figure 1. Evolution of the total atmospheric angular momentum, normalized to its initial value, for the various simulations performed (see section 2.3): 1, reference simulation (bold solid line); 2, same simulation without topography (bold dashed line); 3, simulation with constant $\mathrm{Cp}$ with topography (bold dash-dotted line); 4, simulation with constant $\mathrm{Cp}$ without topography (bold dotted line). Sensitivity simulations are also plotted after the reference run: 5 , no diurnal cycle (thin dashed line); 6 , modified sponge layer (thin dotted line, following closely the reference run); 7 , higher resolution (thin solid line).

48 horizontal grid; (2) one run without the diurnal cycle; (3) sensitivity to upper boundary conditions with a sponge layer damping the horizontal winds and temperature fields toward their zonal average values (no sponge layer at all is unstable and could not be run). This last run is done only for 50 Venus days, as it follows the reference simulation closely.

[33] Other long simulations starting from rest were also performed to analyze the influence of topography and the role of radiative transfer: the same simulation as the reference but without topography ( 350 Venus days), two simulations using a constant $C_{p}$ value $\left(C_{p}=900 \mathrm{~J} \mathrm{~K}^{-1} \mathrm{~kg}^{-1}\right.$, with and without topography, for 250 Venus days), and two simulations using the Newtonian cooling module instead of the full radiative transfer (with and without topography, for 250 Venus days).

\section{Results and Role of Radiative Transfer}

\subsection{Spin-Up Phase and Angular Momentum Conservation}

[34] The evolution of the total angular momentum of the atmosphere, normalized to its initial value $\left(3.47 \times 10^{27} \mathrm{~kg}\right.$ $\mathrm{m}^{2} \mathrm{~s}^{-1}$ ), is shown in Figure 1 for the various simulations mentioned above. The initial value corresponds to an atmosphere with zero zonal wind, i.e., rotating as a solid body at the planet's rotation rate. When the atmosphere is in superrotation, the total angular momentum of the atmosphere is significantly larger than this initial value.

[35] The total angular momentum referred to the rotation axis of the system solid planet and atmosphere is a quantity theoretically conserved in the absence of external (tidal) forces. The total angular momentum of the atmosphere $M$ may therefore only evolve through interactions with the surface: either through pressure effects on the topography (mountain torque), or through boundary layer friction. In a GCM, a sponge layer at the upper boundary may also affect the angular momentum budget.

[36] Superrotation develops in the cloud region in all the long simulations described in the previous section. However, in the deep atmosphere, the zonal wind tends to remain very small compared to observations, and therefore, the total angular momentum pumped into the atmosphere is not greatly increasing.

[37] For the reference simulation, the momentum is still increasing after 350 Venus days. This small increase (around 2\% between 250 and 350 Venus days) may be related to small variations of the zonal wind in the deepest layers, but the overall wind fields are almost identical between 250 and 350 Venus days (displayed below; Figure 6). Without topography, less angular momentum is pumped into the atmosphere, and once the meridional circulation is stabilized, the angular momentum begins to decrease. Again, the small decrease between 250 and 350 days has almost no impact on the overall wind distribution. When a constant $\mathrm{Cp}$ is used, both simulations start pumping angular momentum at similar rates, but without topography, again, the pumping stops after roughly 50 Venus days, and the total angular momentum slightly decreases.

[38] To analyze the angular momentum budget in our simulations, we compute the different terms at high frequency for two Venus days (days 251 and 252). The tendency (temporal variation between two model time steps) on angular momentum is decomposed as

$$
\frac{d M}{d t}=T+F+S+D+\epsilon,
$$

where $M$ is the total angular momentum, $T$ is the total mountain torque, $F$ is the total angular momentum tendency from the boundary layer scheme (friction with the surface), $S$ is the total angular momentum tendency from the sponge layer, $D$ is the residual torque from horizontal dissipation, and $\epsilon$ is the residual variation of angular momentum from the advection scheme. These quantities are computed during the simulation as

$$
M=\int_{V} \rho a \cos \theta u d V+\int_{S} \Omega a^{2} \cos ^{2} \theta \frac{p_{s}}{g} d S,
$$

$$
T=-\int_{S} p_{s} \frac{\partial z_{S}}{\partial \lambda} d S
$$

$$
\begin{aligned}
& F=\int_{V} \rho a \cos \theta\left(\frac{d u}{d t}\right)_{F} d V, \\
& S=\int_{V} \rho a \cos \theta\left(\frac{d u}{d t}\right)_{S} d V, \\
& D=\int_{V} \rho a \cos \theta\left(\frac{d u}{d t}\right)_{D} d V .
\end{aligned}
$$


a)

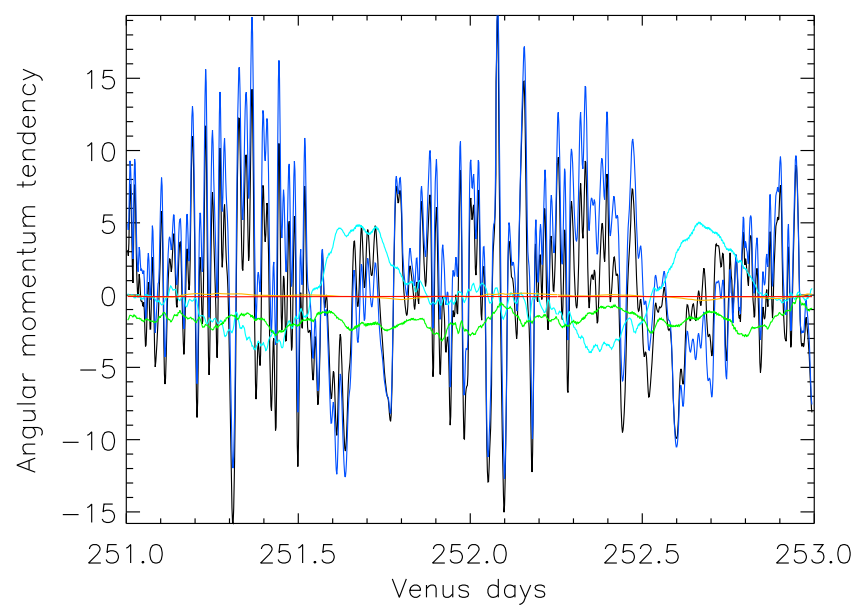

b)

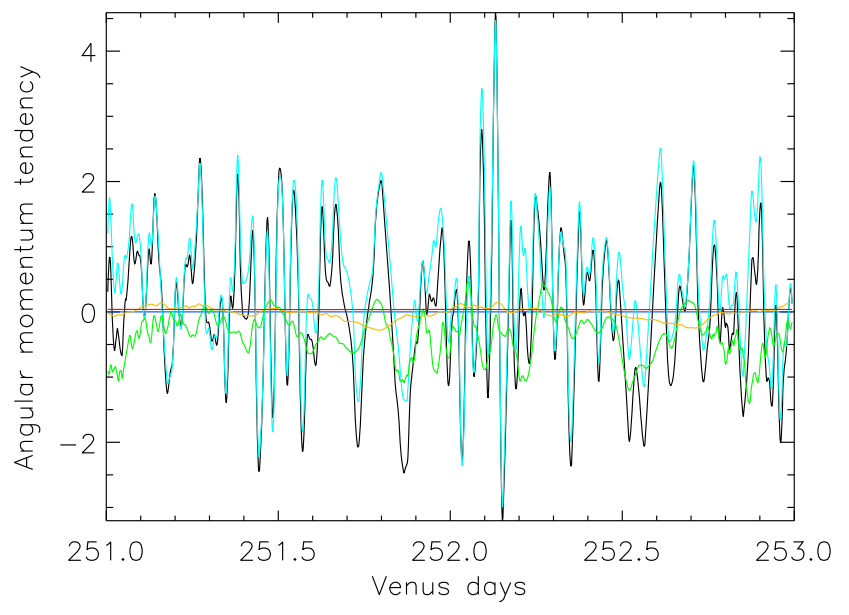

Figure 2. Variations of the total angular momentum tendencies over two Venus days (days 251-252) in the reference simulations (a) with and (b) without topography. Unit is $10^{18} \mathrm{~kg} \mathrm{~m}^{2} \mathrm{~s}^{-2}$. Black, $\frac{d M}{d t}$, temporal derivative of total angular momentum $M$ (computed from successive values of $M$ ); dark blue, $T$, mountain torque; light blue, $F$, surface friction from boundary layer scheme; green, $\epsilon$, residual tendency from advection; orange, $D$, residual tendency from horizontal dissipation; red, $S$, sponge layer.

In these equations, $\int_{V} d V$ is the integral over the volume atmosphere, $\int_{S} d S$ is the integral over the surface of Venus, $\rho$ is the density, $\lambda$ is the longitude, $\theta$ is the latitude, $a$ is the radius of Venus, $\Omega$ is the angular velocity of Venus' rotation, $z_{s}$ is the topographic height, $p_{s}$ is the surface pressure, $u$ is the zonal wind, and $\left(\frac{d u}{d t}\right)_{F, S, D}$ are the different tendencies computed in the GCM modules. $\epsilon$ appears as the difference $\frac{d M}{d t}-(T+F+D+S)$. $D$ and $\epsilon$ should theoretically be equal to zero. These different terms are plotted as a function of time for two Venus days in Figure 2 (days 251 and 252 of the simulations), for the reference simulation and the simulation without topography. Their average values over these two days are listed in Table 3. These two days are quite representative of any time during the simulations. The average value of $\frac{d M}{d t}$ over these two days is equal to the trends seen in Figure 1 after 250 Venus days.
[39] From Figure 2, it appears that $D$ and $S$ are quite stable, and very small compared to the average value of $T+$ $F$, which represents the total interaction between the atmosphere and the surface. Though this is a critical point on which a particular effort was put in the LMDZ GCM, the conservation of the atmospheric angular momentum during advection is not exact in the numerical model: the $\epsilon$ term (residual after advection) is not zero, indicating a minor problem of angular momentum conservation. This $\epsilon$ residual is also quite stable, and small compared to the amplitude of the $T+F$ variations, though its absolute value is comparable to the average value of $T+F$ after 250 Venus days. However, $\epsilon$ appears to be always negative, which means that during advection, the model tends to lose numerically some of the atmospheric angular momentum. Therefore, this leak can not be an artificial source of angular momentum that would drive superrotation, though it may slightly affect the spin-up rate. The angular momentum pumping into the atmosphere occurs through the $T+F$ interaction with the surface, which decreases during spin-up until it approximately balances the $\epsilon$ numerical leak.

[40] An interesting feature to note in Figure 2a is the diurnal cycle of $T$ and $F$. Depending on the relative positions of the subsolar point and the topographic features, the surface winds organize so that the total surface friction either takes momentum from the surface $(F>0)$ or give it back $(F<0)$. The mountain torque follows a similar cycle (though anticorrelated with the total surface friction), with much larger amplitude, higher frequency variations, and positive average value. This diurnal cycle induces diurnal variations of the total atmospheric angular momentum through exchanges with the solid planet, and therefore variations of the planet's rotation rate [Karatekin et al., 2009]. This length of day (LOD) variation, which is the variation in Venus' rotation rate, can be computed using

$$
\Delta \mathrm{LOD}=\frac{\Delta M_{\mathrm{atm}}}{M_{\mathrm{Venus}}} \times \mathrm{LOD},
$$

where $M_{\text {Venus }}=1.87 \times 10^{31} \mathrm{~kg} \mathrm{~m}^{2} \mathrm{~s}^{-1}$ is the angular momentum of the solid body, LOD $=2.1 \times 10^{7} \mathrm{~s}$ is the rotation period of Venus, and $\Delta M_{\mathrm{atm}}$ is the variation of the total atmospheric angular momentum over a period of 117 Earth days, which is around $7 \times 10^{24} \mathrm{~kg} \mathrm{~m}^{2} \mathrm{~s}^{-1}$ in this simulation. This yields $\Delta \mathrm{LOD} \simeq 7.9 \mathrm{~s}$.

\subsection{Zonally Averaged Meridional Structure}

\subsubsection{Temperature and Stability}

[41] Available observations of the temperature structure can be found in the work by Seiff [1983] and Seiff et al.

Table 3. Averaged Values of the Different Terms in Equation (12) Over the Two Venus Days Shown in Figure $2^{\mathrm{a}}$

\begin{tabular}{lcc}
\hline & With Topography & Without Topography \\
\hline$d M / d t$ & 0.12 & 0.03 \\
$T$ & 1.95 & 0. \\
$F$ & 0.08 & 0.39 \\
$S$ & -0.07 & -0.03 \\
$D$ & -0.10 & 0.04 \\
$\epsilon$ & -1.74 & -0.37 \\
\hline
\end{tabular}

${ }^{\mathrm{a}}$ Unit is $10^{18} \mathrm{~kg} \mathrm{~m}^{2} \mathrm{~s}^{-2}$. 


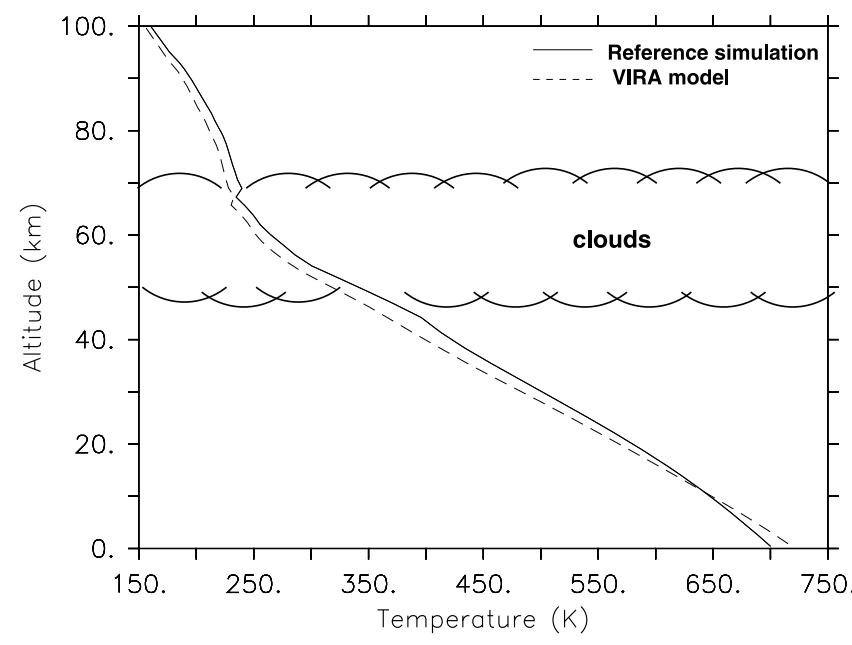

Figure 3. Vertical temperature profile (solid line) obtained after 350 Venus days in the reference simulation, averaged over longitudes, latitudes, and the last two Venus days of the simulation. It is compared to the initial temperature profile, close to the VIRA model (dashed line). Cloud boundaries are sketched for indication.

[1985] (Pioneer Venus), Zasova et al. [2007] (Venera missions), and Grassi et al. [2008] and Tellmann et al. [2009] for recent Venus Express data. The vertical structure of the temperature averaged over time (the last two days of the simulation), longitudes and latitudes is shown in Figure 3. It is close to the VIRA model [Seiff et al., 1985], except in the deepest $10-15 \mathrm{~km}$, where temperatures are lower than observed, and the related static stability is much larger than observed. This discrepancy may be related to the uncertainties that are present in the spectroscopic data sets used as inputs for the NER matrix computations (see dis- cussions by Eymet et al. [2009]). The static stability profile is shown in Figure 4. It may be compared to the stability profiles deduced from the Pioneer Venus data [Schubert, 1983], from the Vega 2 entry probe (also reproduced in Figure 4 [Zasova et al., 2007]), and from the $\mathrm{VeRa} / \mathrm{Venus}$ Express data [Tellmann et al., 2009]. The dominant features are the convective region located in the lower and middle clouds (the region with very small stability around $50 \mathrm{~km}$ altitude, discussed in section 3.3), the stable layer below the clouds, and the low-stability deep atmosphere (below $30 \mathrm{~km}$ altitude).

[42] Latitudinal variations of the temperature field are shown in Figure 5, where the difference between the zonally and temporally (last two Venus days of the simulation) averaged temperature and its latitudinal mean is plotted. Below $55 \mathrm{~km}$, the temperature contrasts between equator and poles are very small, less than 1 to $2 \mathrm{~K}$. This is much lower than variations seen in available observations, that may reach $20-30 \mathrm{~K}$ at $50 \mathrm{~km}$ altitude [Seiff et al., 1980; Sromovsky et al., 1985; Zasova et al., 2007; Tellmann et al., 2009].

[43] In the upper cloud $(60-70 \mathrm{~km})$, the observations show the presence of a cold collar, around $40 \mathrm{~K}$ colder than equator, located at latitudes around $60^{\circ}-75^{\circ}$, with a hot core above the pole. The temperature contrast modeled in this region is only around $10 \mathrm{~K}$, and no hot polar core is obtained. This discrepancy may be explained by the fact that the cloud structure observed in this region also has characteristics different from the equator, with a lower cloud top and different cloud particle properties [Titov et al., 2008; Wilson et al., 2008]. These cloud structure variations are not yet implemented in the model, and since there is an interaction between temperature structure and cloud structure, this lack of cloud structure variations in the model is probably related to the discrepancy in the latitudinal temperature contrast.

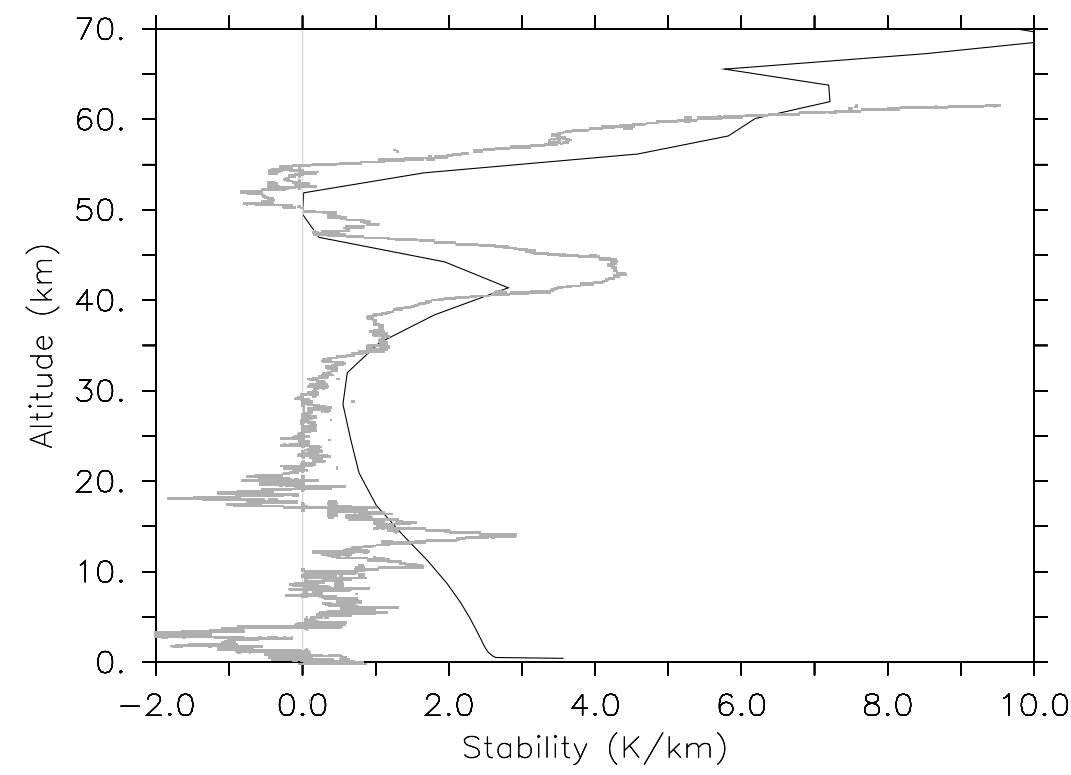

Figure 4. Vertical stability profile obtained after 350 Venus days in the reference simulation, averaged over longitudes, latitudes, and the last two Venus days of the simulation (black line). Data obtained from the Vega 2 entry probe (taken from Zasova et al. [2007]) are shown for comparison (gray line). 


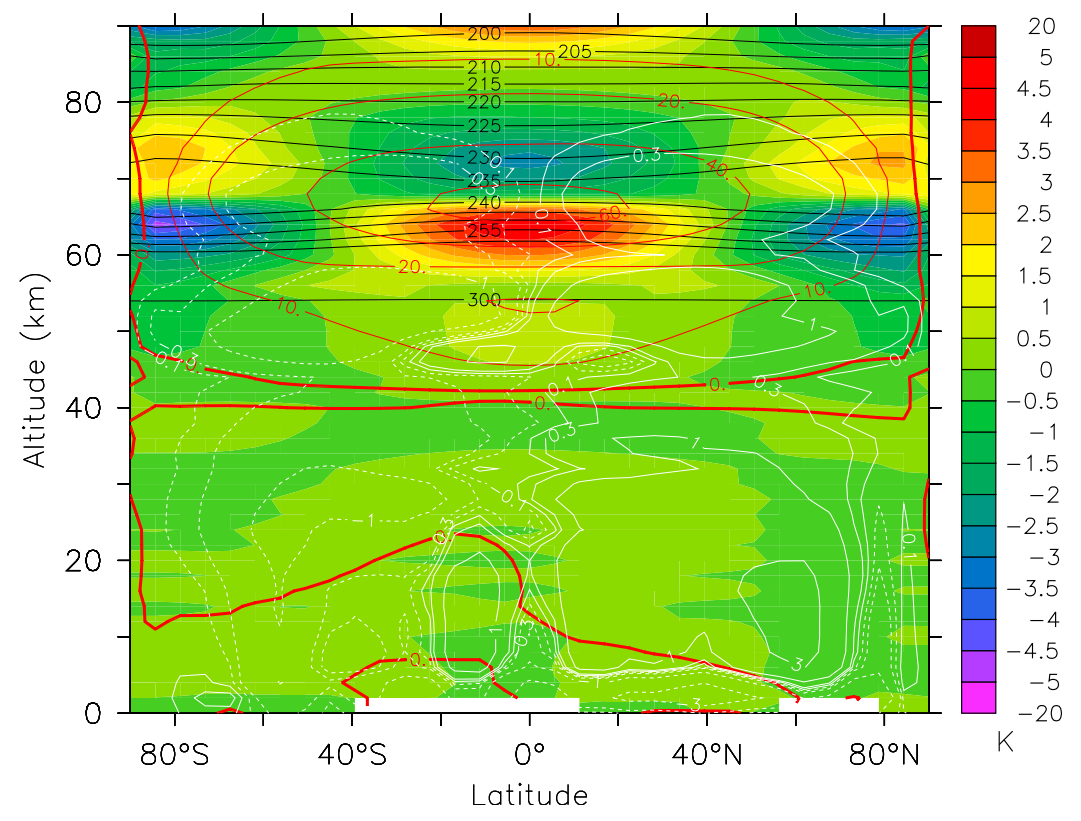

Figure 5. Latitudinal temperature contrasts $(K)$ for the reference simulation: difference between the zonally and temporally (last two Venus days of the simulation) averaged temperature and its latitudinal mean. Contours show the mean zonal wind (red, $\mathrm{m} \mathrm{s}^{-1}$ ), the mean temperature field (black, $\mathrm{K}$ ), and the mean meridional circulation (mass stream function, white, $10^{9} \mathrm{~kg} \mathrm{~s}^{-1}$ ).

[44] Above roughly $70 \mathrm{~km}$ altitude, the latitudinal gradient of temperature reverses, with warmer temperatures above the pole. This is consistent with observations, and with the decrease of zonal wind speed (see below). It is related to adiabatic heating and cooling in the upper region of the mesospheric equator-to-pole meridional cells.

\subsubsection{Zonal Wind}

[45] Using realistic radiative transfer instead of the simpler temperature forcing used in other Venus GCMs results in significant differences in the mean zonal wind field, but also in the mean meridional circulation, as seen in Figure 6.

[46] Figures $6 \mathrm{c}$ and $6 \mathrm{~d}$ illustrate the circulations obtained with the simplified radiative forcing after 250 Venus days, to compare to previous GCMs using similar radiative parameterization [Yamamoto and Takahashi, 2003, 2004, 2006; Lee, 2006; Lee et al., 2005, 2007; Herrnstein and Dowling, 2007; Hollingsworth et al., 2007]. The results are mostly similar to these previous studies. These simulations are not discussed in detail here, because the goal of this paper is to focus on the simulations with realistic radiative transfer. However, we wanted to illustrate how the different radiative forcings result in different circulations. Therefore, the circulations obtained with simplified radiation scheme are briefly described below.

[47] Maximum zonal winds are obtained in the cloud deck, with high-latitude jets located near $60^{\circ}-70^{\circ}$ latitude, and around $50 \mathrm{~km}$ altitude, lower than the level of maximum temperature forcing (around 55-60 km altitude). A secondary maximum is located at the equator, close to the top of the model. The mean meridional circulation consists essentially of two large Hadley cells, with ascending motions at the equator, and subsiding motions over polar regions, covering all altitudes from the surface up to the cloud regions above $60 \mathrm{~km}$ altitude. In their work, Herrnstein and Dowling [2007] point out that taking into account the topography reduces the zonal wind peak. This is not the case here. Both the zonal wind peak amplitudes and the overall amplitude of the superrotation is higher with topography in our simulations.

[48] The atmospheric circulation is different when a realistic radiative transfer is used. Zonal wind and mean meridional circulation are shown in Figures 6a (with topography) and $6 \mathrm{~b}$ (without). We emphasize that the circulation described below is the mean meridional circulation, averaged over longitude and time. The day and night circulations are different, and will be discussed in future work.

[49] In these simulations, the modeled Venus atmosphere appears to be vertically divided into three different regions: (1) the deep troposphere, from surface to the base of the clouds (around $45 \mathrm{~km}$ altitude), where the influence of the topography over the meridional circulation is clearly visible, and the zonal wind is very small; (2) the lower and middle cloud region, from 45 to $60 \mathrm{~km}$ altitude, dominated by two thermally direct cells, where the zonal winds start increasing; and (3) the upper cloud and mesosphere, above $60 \mathrm{~km}$ altitude, where the zonal winds are maximum, with a dominant role played by the thermal tides. These three different regions are detailed in the following subsections.

[50] Because the zonal wind and temperature mean meridional fields are linked together through cyclostrophic equilibrium (which is approximately verified from the cloud base upward in the model), the three regions in the vertical structure are also visible in the mean latitudinal temperature contrast (i.e. the difference between the zonal and temporal averaged temperature and its latitudinal mean, Figure 5). The strongest contrasts are seen in the upper cloud and mesosphere, where the peak of the zonal wind is located. The relation between the vertical variations of the zonal wind and the latitudinal contrasts in the temperature field is clearly visible in this map. 
a)

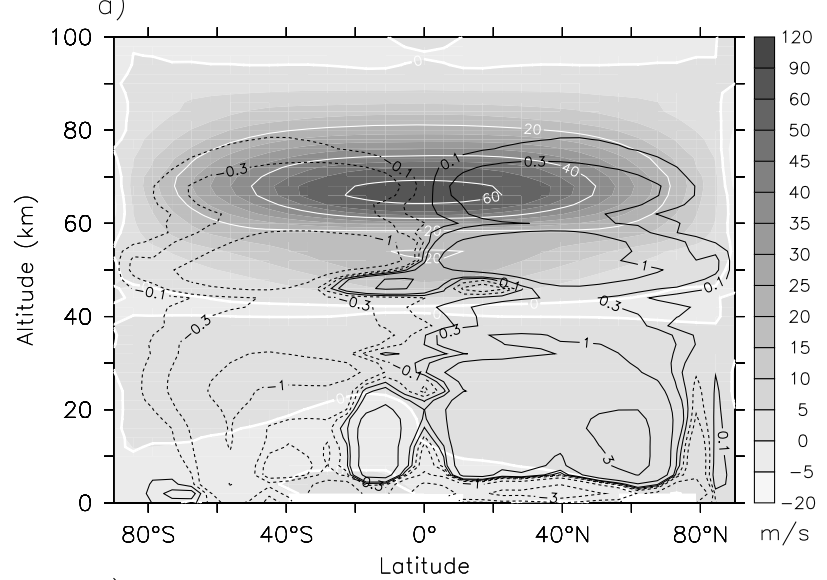

c)

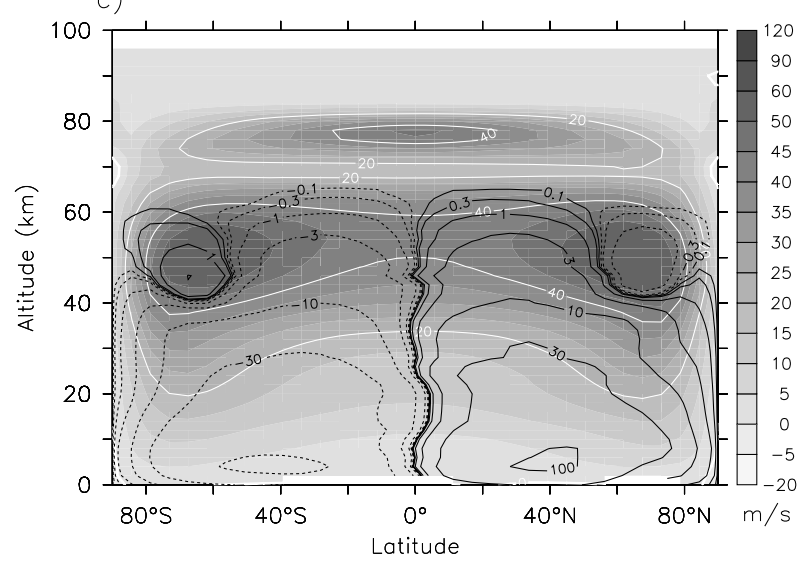

b)

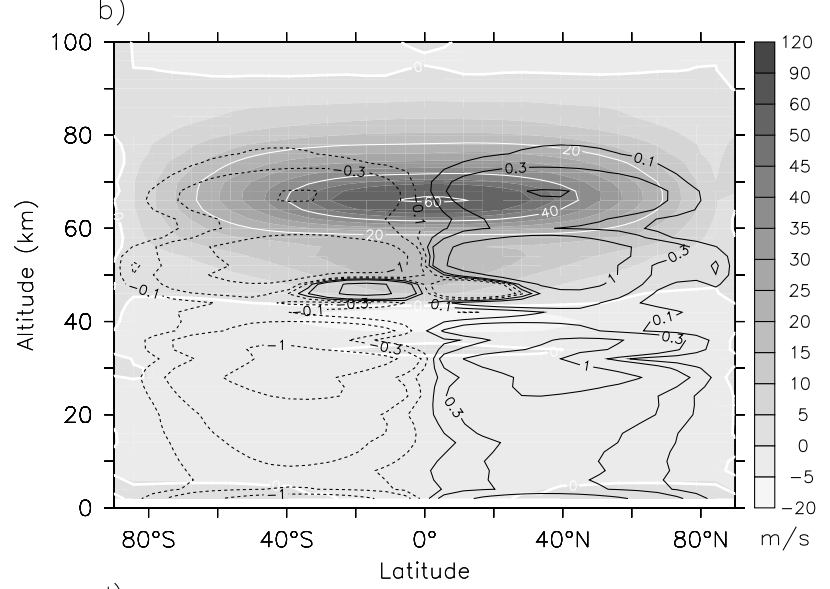

d)

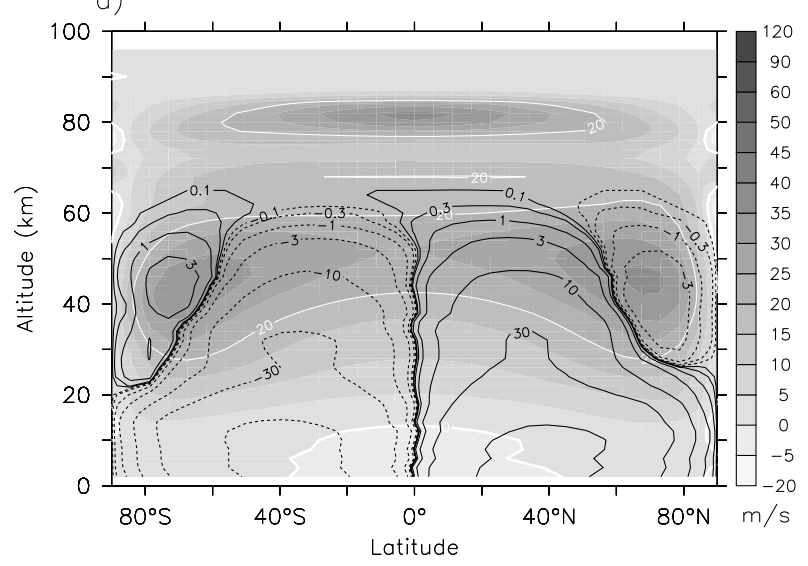

Figure 6. Mean zonal wind $\left(\mathrm{m} \mathrm{s}^{-1}\right)$ and stream function (black contours, $10^{9} \mathrm{~kg} \mathrm{~s}^{-1}$ ) after 350 Venus days for (a) reference simulation with full radiative transfer and topography, (b) same as Figure 6a but without topography, (c) simulation with Newtonian cooling forcing and topography, and (d) same as Figure $6 \mathrm{c}$ but without topography. Averages are done over the last 2 Venus days of each simulation and over longitudes.

[51] The modeled wind field does not fit the available observed vertical and latitudinal profiles of the zonal wind field [Schubert, 1983; Gierasch et al., 1997; Limaye, 2007; Peralta et al., 2007; Sánchez-Lavega et al., 2008]. The observed and modeled vertical profiles of the equatorial zonal wind are shown in Figure 7. The measured winds below $40 \mathrm{~km}$ altitude are increasing steadily with altitude. This is not the case in the simulations. The amplitude of the zonal wind peak, though located at the right altitude, appears to be approximately half of the observed value.

[52] When we look at latitudinal variations of the zonal wind within the cloud layers, the observed profile at the cloud top is roughly constant up to $50^{\circ}-60^{\circ}$ latitude, which is not the case in the simulation.

\subsection{Lower and Middle Clouds: From 45 to $60 \mathrm{~km}$}

[53] This region covers the dominant thermally direct circulation obtained in the simulation. This structure is closely related to the mean radiative forcing, and to the energy redistribution in this region. The radiative temperature tendencies are shown in Figure 8: both solar heating rate and infrared cooling rate are added, giving the total temperature variation rate due to the radiative scheme. The average is performed over the last two Venus days of the simulation, and over longitudes. The solar radiation yields a maximum heating over equatorial regions, above roughly $55 \mathrm{~km}$ altitude. The meridional circulation consists of two equator-to-pole cells, with ascending motions at the equator, subsiding motions over the poles, an upper poleward branch in the middle clouds, which redistributes energy poleward, and a lower equatorward branch in the lower clouds. The zonal wind in this region peaks at the equator, with smaller values than expected from observations. The angular momentum transport associated with this meridional circulation will be discussed in section 4 .

[54] The patterns between 45 and $55 \mathrm{~km}$ are due to the longwave infrared emission and absorption: a maximum cooling between 50 and $55 \mathrm{~km}$ altitude, dominant above polar regions though it covers all latitudes, and a secondary maximum heating at the base of the clouds (around $47 \mathrm{~km}$ altitude over all latitudes in this simulation) where absorption by the clouds of thermal infrared radiation coming from below dominates the energy exchanges. This structure tends to destabilize this atmospheric region, since it tends to produce superadiabatic temperature gradients. Therefore, convection is obtained between 45 and $57 \mathrm{~km}$, as seen in Figure 8 , but also in the stability profile (Figure 4), in close 


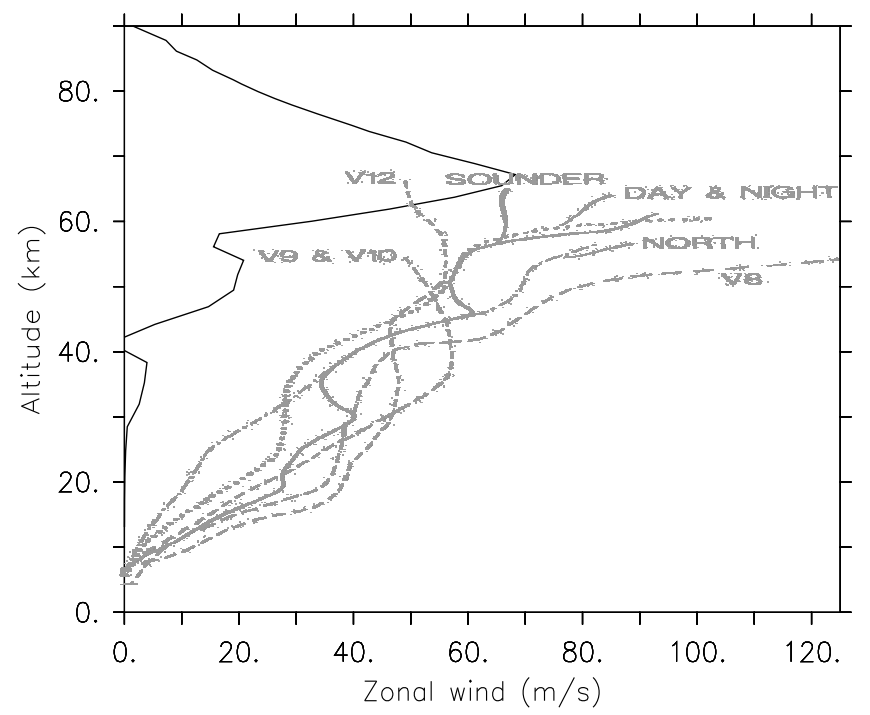

Figure 7. Vertical profile of the zonal wind at equator obtained after 350 Venus days in the reference simulation, averaged over longitudes and the last two Venus days of the simulation (black line). Data obtained from the Venera and Pioneer Venus missions (taken from Schubert [1983]) are shown for comparison (gray).

agreement with the observed stability profile in this region [Schubert, 1983; Tellmann et al., 2009].

[55] This pattern is also illustrated in Figure 9, where the thermal infrared radiative budget (i.e. the net power received by a model cell per unit horizontal area from the infrared radiative scheme) is shown as a function of altitude, and as a function of wavelength (from 1.7 to $250 \mu \mathrm{m}$ ). This budget is computed with the $\xi$ matrix used in the GCM (for a surface pressure of 92 bars), and with the averaged temperature profile at the end of the reference simulation, presented in Figure 3.

[56] This vertical profile shows a maximum heating at the base of the clouds, and a maximum cooling in the middle of the cloud layers, between 50 and $55 \mathrm{~km}$ altitude (pattern already seen in previous radiative transfer studies [Crisp and Titov, 1997]). A second maximum cooling is also computed at the top of the clouds. The structure of the cooling within the whole cloud region is sensitive to the cloud model used in the radiative transfer. The heating at the base of the clouds is done from the hot troposphere below, through infrared windows between 3 and $7 \mu \mathrm{m}$, while cooling in the clouds is due to exchanges of energy between the cloud layers and space, mainly through windows around $10 \mu \mathrm{m}$, and a window ranging from roughly 20 to $30 \mu \mathrm{m}$.

\subsection{Upper Cloud and Mesosphere}

[57] Above $60 \mathrm{~km}$, in the mesosphere, the zonal winds reach maximum values around $70 \mathrm{~km}$ altitude, with a peak centered on the equator. The meridional circulation is organized in weak Hadley cells.

[58] The amplification of the equatorial zonal wind in this region, as well as in the other cloud region discussed in the previous subsection, is related to the diurnal tides. This is demonstrated below, in section 4. The latitudinal distribution of zonal wind in this region may then result from an equilibrium between the impact of thermal tides, and the angular momentum transport by the meridional circulation. The intensity of this meridional transport is correlated with the meridional transport of energy, and therefore with the radiative forcing. A better representation of clouds in the radiative scheme, taking into account the latitudinal varia-

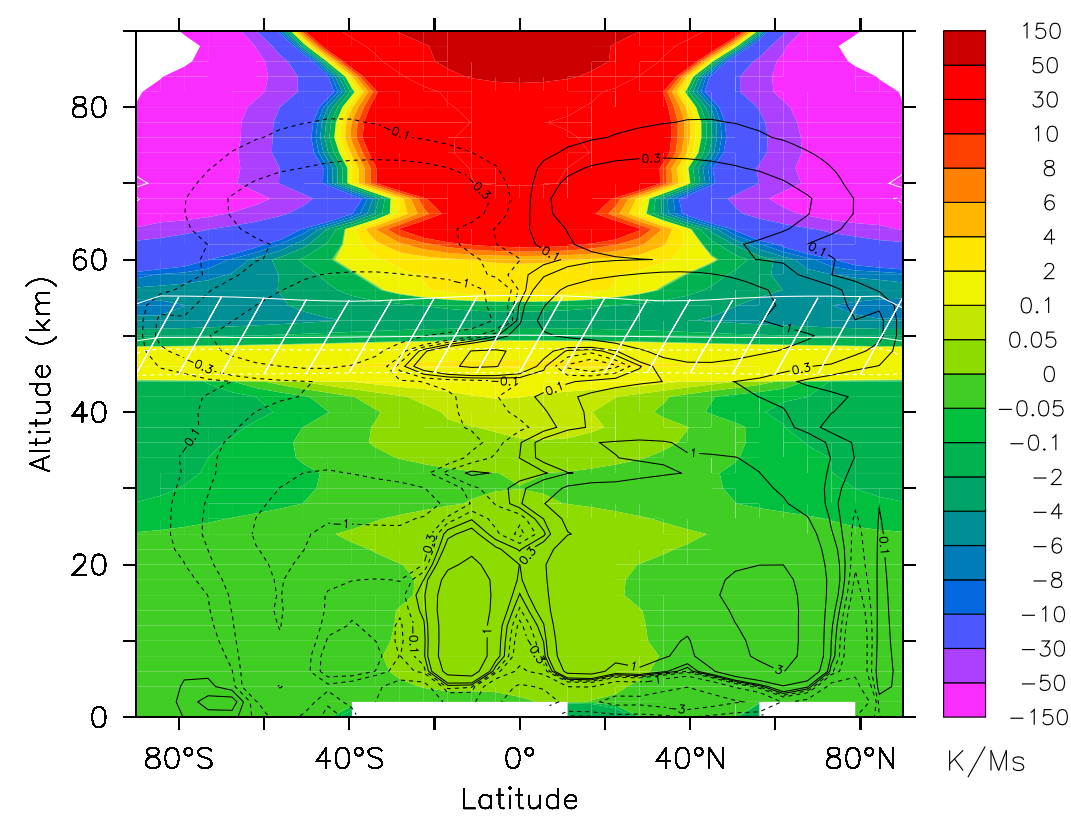

Figure 8. Radiative temperature tendency (sum of solar and infrared temperature tendencies), averaged over the last two days of the simulation (unit is $10^{-6} \mathrm{~K} \mathrm{~s}^{-1}$ ). Contours show the temperature tendency due to convective adjustment (white, with the dominant convective region highlighted by the dashed area) and mass stream function (black, $10^{9} \mathrm{~kg} \mathrm{~s}^{-1}$ ). 
a)

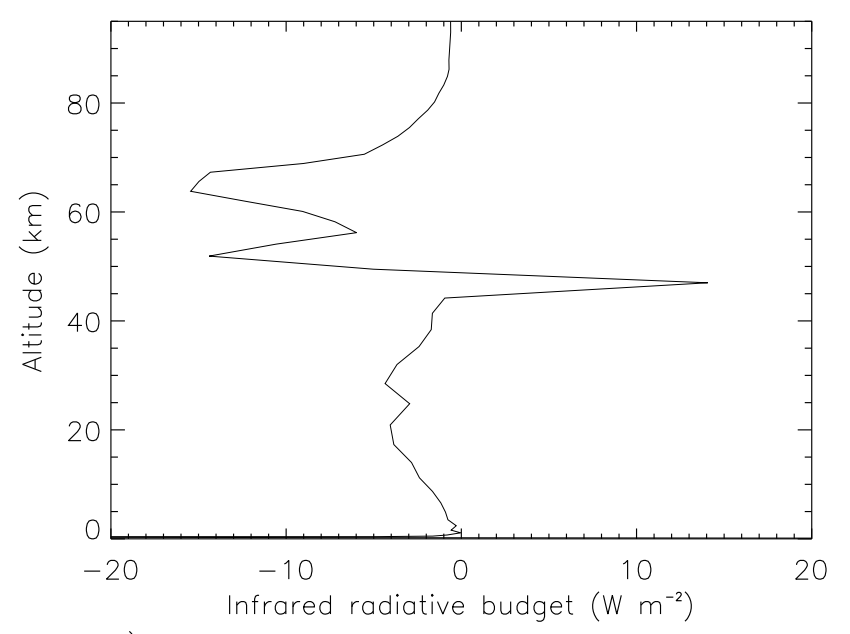

b)

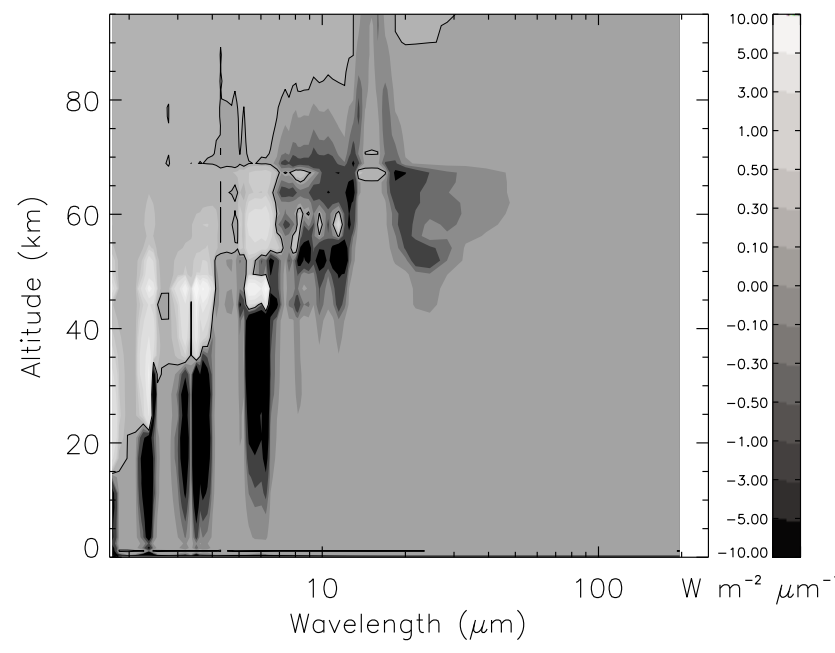

Figure 9. (a) Vertical profile of the radiative budget integrated over infrared wavelengths (from 1.7 to $250 \mu \mathrm{m}$ ), computed using the equatorial $\xi$ matrix and the averaged temperature profile at the end of the reference simulation, presented in Figure 3. (b) The same vertical profile of thermal IR radiative budget but shown as a function of wavelength (unit is $\mathrm{W} \mathrm{m}^{-2} \mu \mathrm{m}^{-1}$ ).

tions in the cloud distribution, may improve both the latitudinal temperature contrast, and the balance between meridional circulation and thermal tides in the angular momentum transport.

\subsection{Deep Troposphere: From 0 to $45 \mathrm{~km}$}

[59] Between the surface and the cloud base, the meridional circulation is significantly affected by topography. Without topography, the mean meridional circulation is symmetric with respect to the equator, and is organized as two Hadley cells. When topography is taken into account, though the two dominant cells are still visible, the symmetry is broken, and small cells are present, in particular an indirect cell close to the surface in the northern low to middle latitudes, between the main topographic features (Figure 6a). Again, this is a zonally averaged view of the meridional circulation, and its variation with local time will be studied in detail in future work.

[60] Near $30 \mathrm{~km}$ altitude, there is a region with a clear minimum in the stability profile, in agreement with observations (see Figure 4). This feature is related to a secondary maximum of cooling at these altitudes (Figure 8), mainly occurring in the 3-7 $\mu \mathrm{m}$ window (Figure 9b).

[61] Below roughly $20 \mathrm{~km}$ altitude, the stability becomes too high in the model, compared to observations, and the temperature is lower than observed.

[62] The zonal wind remains very small from the surface up to the cloud base, which is in strong disagreement with the observations (Figure 7). This is consistent with the total angular momentum remaining much smaller than expected from available measurements. This problem is not understood, but this result was also obtained by Ikeda et al. [2007] with a similar general circulation model, and a very different (but self-consistent) radiative scheme: superrotation develops in their model within and above the clouds, but no significant zonal winds appear under the clouds. In our GCM, this discrepancy may possibly be related to the problem of the atmosphere above the surface being too stable, or to the fact that latitudinal variations of the cloud structure is not taken into account. Some preliminary tests have also indicated that this problem may be related to the parameterization chosen for the vertical diffusion and surface drag coefficients, both computed in the boundary layer module. A complete study of the impact of these parameters on the deep atmospheric circulation will be reported in a future work. Another hypothesis, proposed by Ikeda et al. [2007], is the possible role of nonorographic gravity waves generated near the surface. Using a parameterization for such nonorographic gravity waves, these authors showed that the zonal wind in the lower atmosphere is increasing, and the overall profile is much closer to observations. In their model, gravity waves generated close to the surface with positive phase speeds encounter critical levels before reaching the cloud base, depositing angular momentum below the clouds and accelerating the zonal mean flow. On the contrary, gravity waves with negative phase speed reach the high atmosphere above the clouds, decelerating the flow in this region.

\subsection{Sensitivity Studies}

[63] Figure 10 shows the sensitivity of the modeled wind field to some parameters. When a constant $C_{p}$ is used (Figure 10a), the modeled circulation is quite similar to the reference simulation after 250 Venus days. The use of a different $C_{p}$ profile affects mainly the temperature profile (not shown). The separation between the deep atmosphere below the clouds and the lower cloud appears to be more pronounced, and the zonal wind peak less intense.

[64] The use of a different sponge layer (Figure 10b) for 50 Venus days from the 250 Venus day state of the reference simulation (see section 2.3) do not seem to affect the circulation significantly.

[65] When using a finer spatial grid (Figures 10c and 10d) for up to 100 additional Venus days after the reference simulation taken at 250 Venus days (see section 2.3), the zonal wind field distribution does not appear to be significantly changed, though the total angular momentum slightly decreases (as seen in Figure 1). However, the higher spatial 
a)

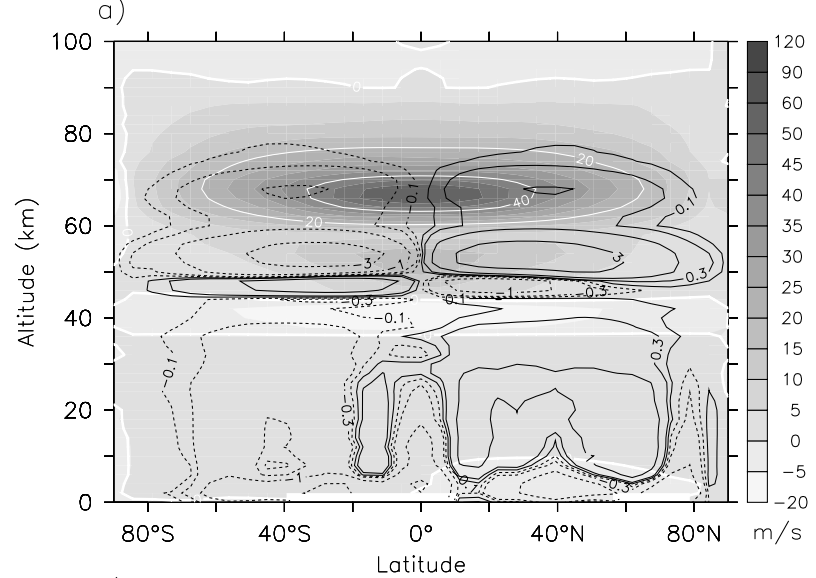

c)

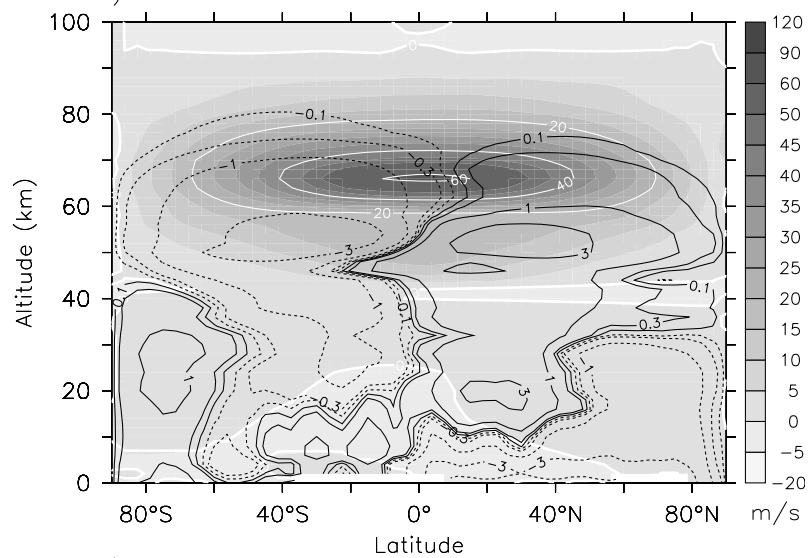

e)

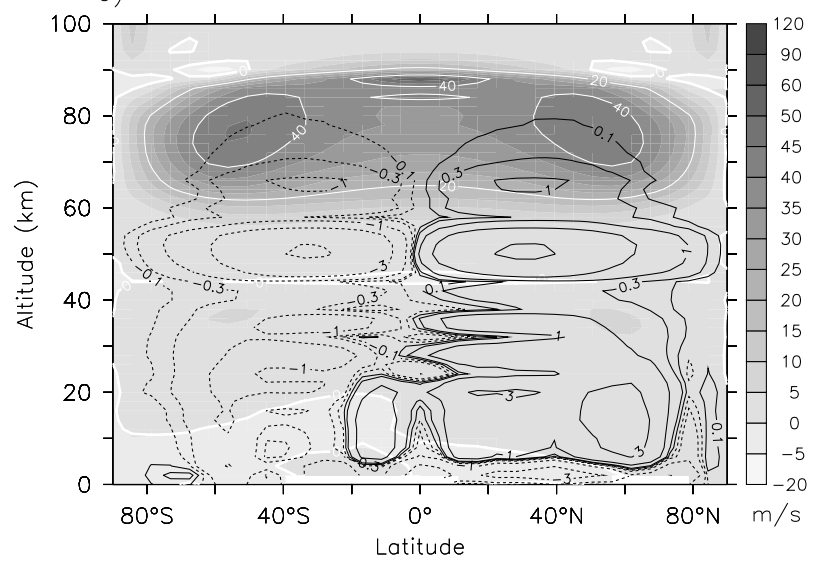

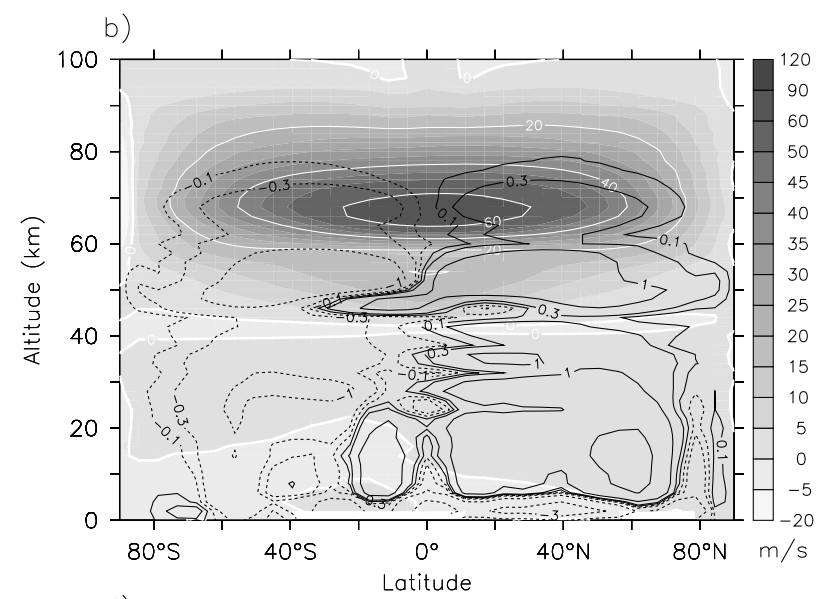
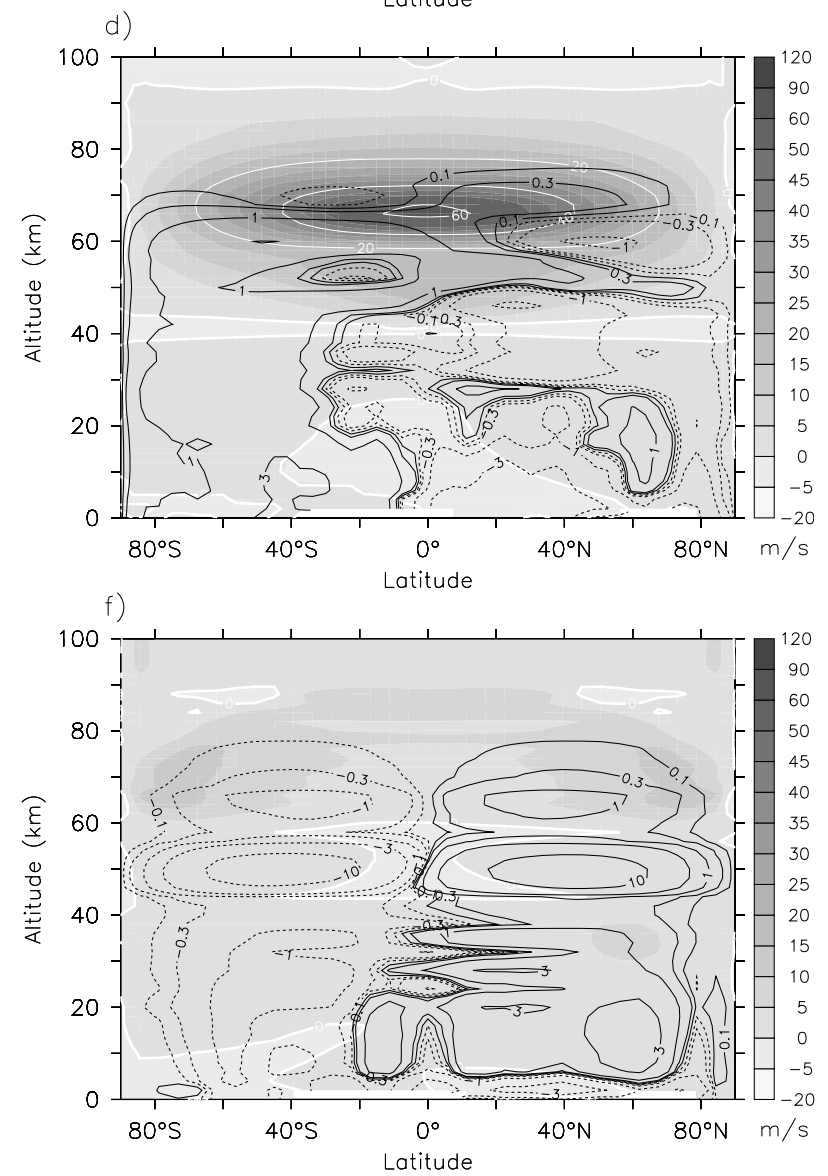

Figure 10. Mean zonal wind $\left(\mathrm{m} \mathrm{s}^{-1}\right.$ ) and stream function (black contours, $10^{9} \mathrm{~kg} \mathrm{~s}^{-1}$ ) for sensitivity studies (to be compared to Figure 6a): (a) with constant $C_{p}$, full radiative transfer and topography (after 250 Venus days); (b) with modified sponge layer (50 additional Venus days after the reference simulation taken at day 250); with higher horizontal resolution $(64 \times 48)$ after (c) 50 and (d) 100 additional Venus days after the reference simulation taken at day 250; and without diurnal cycle after (e) 50 and (f) 100 additional Venus days after the reference simulation taken at day 250. Averages are done over the last 2 Venus days of simulations and over longitudes.

resolution affects the averaged meridional stream function, mainly below $40 \mathrm{~km}$ altitude where the topography plays a significant role, as discussed above. A more extensive evaluation of the sensitivity to resolution should be performed in the future, but as far as the temperature, zonal wind and angular momentum are concerned, this sensitivity test indicates a very minor impact.
[66] The most significant effect is clearly seen in Figures 10e and 10f, when the diurnal cycle is omitted for 100 additional Venus days after the 250 Venus day reference simulation. In the lower and middle clouds, the zonal winds are reduced to very low values, and even negative values around the equator (Figure 10f). In the upper clouds and above, the zonal winds around the equator are significantly less than in the reference 
simulation, with high latitude jets appearing and then fading away. The evolution after 50, then 100 Venus days clearly indicates that the superrotation in the cloud region is no longer maintained, though the situation in the deeper atmosphere appears to be less affected (see also Figure 1).

[67] This is a clear signature of the influence of thermal tides in the distribution of angular momentum within and above the cloud region. This role will be discussed in more details in section 4.2.

[68] This structure of the zonal wind field obtained in the simulation without diurnal cycle has some similarities with the simulations using a simplified temperature forcing, though the jets obtained are located much lower, and stable, in the latter case. In the simplified forcing case, the diurnal cycle is also not included. Lee [2006] tried including a diurnal cycle in the simplified forcing, but he did not get significant influence on the zonal wind field, though the diurnal tides did play a role in the angular momentum transport.

\section{Superrotation Mechanism}

[69] Though this peculiar circulation has been studied for thirty years, and despite available observations of Venus' atmospheric dynamics, the mechanisms controlling the superrotation of Venus' atmosphere are still not fully understood. The GRW mechanism [Gierasch, 1975; Rossow and Williams, 1979] has been able to explain Titan's atmospheric superrotation in the most evolved Titan GCM to date [Hourdin et al., 1995, 2004; Rannou et al., 2004], and in previous simplified GCMs developed for Venus [Young and Pollack, 1977; Del Genio et al., 1993; Yamamoto and Takahashi, 2003; Lee et al., 2005; Herrnstein and Dowling, 2007; Hollingsworth et al., 2007], but some studies have also emphasized that thermal tides have a significant effect [Pechman and Ingersoll, 1984; Fels, 1986; Leovy and Baker, 1987; Hou et al., 1990; Newman and Leovy, 1992; Takagi and Matsuda, 2007]. The zonally averaged transport of angular momentum in our new simulations of Venus' atmospheric dynamics is detailed in this section. Contributions of mean meridional circulation (MMC), transient waves and stationary waves are separated, after averaging over 4 Venus days (computed with high-frequency outputs). The total meridional transport $[\overline{v q}]$ of a variable $q$ can be decomposed as

$$
[\overline{v q}]=[\bar{v}][\bar{q}]+\left[\overline{v^{*}}\right]\left[\overline{q^{*}}\right]+\left[\overline{v^{\prime} q^{\prime}}\right]
$$

where $[q]$ is the zonal mean, $\bar{q}$ the temporal average, $q^{*}=$ $q-[q]$, and $q^{\prime}=q-\bar{q} .[\bar{v}][\bar{q}]$ is the MMC contribution, $\left[v^{*}\right]$ $\left[q^{*}\right]$ is the contribution from stationary waves, and $\left[\overline{v^{\prime} q^{\prime}}\right]$ is the contributions from transients waves. The same equation applies for vertical transport of the variable $q$, using the vertical wind $w$ (in $\mathrm{Pa} / \mathrm{s}$ ) instead of the meridional wind $v$. The stationary waves component appears to be negligible (despite topography), and the respective role of MMC and transients will be discussed below.

[70] In the following discussion, two simulations are used: the reference simulation (after 350 Venus days), and the sensitivity simulation done without diurnal cycle, taken 50 Venus days after the 250 Venus day state of the reference simulation, which will be referred to as NDC for no diurnal cycle. From these simulations, we compute the relative angular momentum of a given cell (mass $\delta m$ ) at latitude $\phi$ from the zonal wind $u: \delta M=\delta m \times u \times a \cos \phi$, where $a$ is Venus' radius.

\subsection{Angular Momentum Transport Without Diurnal Cycle}

[71] The zonally averaged vertical and horizontal transports of angular momentum by the circulation are shown in Figures 11 and 12 for the NDC simulation. We used the simulation after 50 Venus days only because the zonal circulation is not maintained, and the transport of angular momentum after 100 Venus days, though qualitatively similar to the one discussed here, is much weaker.

[72] Figures 11 and 12 are typical of the GRW mechanism [Gierasch, 1975; Rossow and Williams, 1979]. The mean meridional circulation transports angular momentum upward (Figure 11a), and toward the poles (Figure 12a). This transport is compensated by the transport by waves (transients), which bring back angular momentum horizontally toward the equator, and vertically downward. An analysis of these waves is given in section 4.3.

[73] To go deeper in the analysis of the transport, the vertical transport is dissociated in relevant regions in Figure 11: the middle- and low-latitude region between $50^{\circ} \mathrm{N}$ and $50^{\circ} \mathrm{S}$ (Figure 11c) and the two polar regions (north in Figure 11b and south in Figure 11d). The same is done for the horizontal transport in Figure 12: the region above $60 \mathrm{~km}$ altitude (Figure 12b), the region below $42 \mathrm{~km}$ altitude (Figure 12d), and the region in between (Figure 12c).

[74] In the vertical direction, Figure 11 shows that the mean meridional circulation transports upward the angular momentum at low to midlatitudes, while the downward transport is done by the mean meridional circulation in polar regions, and by transients at low to midlatitudes. In the horizontal direction, the transport is dominant in the region corresponding roughly to the lower and middle clouds (42 to $60 \mathrm{~km}$ altitude), as well as in the region corresponding to the upper clouds and above (above $60 \mathrm{~km}$ altitude). In each region, the poleward transport by each of the Hadley cells is compensated by an equatorward transport by waves generated in these regions.

[75] The resulting distribution of zonal wind (Figure 10e) is consistent with the GRW mechanism. It exhibits a highlatitude peak in each hemisphere, which is favorable for barotropic instabilities. This may then produce waves that will induce the stabilizing equatorward transport of angular momentum [Luz and Hourdin, 2003]. However, it appears that this mechanism is not stable here, as seen in Figure 10f. The jets are fading away, indicating that the mean meridional circulation at low latitudes (where the zonal wind has decreased a lot) is not injecting enough angular momentum above the clouds to maintain the jets.

[76] Together with the transport of angular momentum by the meridional and vertical winds discussed above, angular momentum is also transported in the physical parameterizations through the boundary layer and advection schemes. These terms are not included in Figures 11 and 12. The transport by the boundary layer scheme compensates the residual transport, and brings back angular momentum from polar regions (where it is given back to the surface) to 
a)
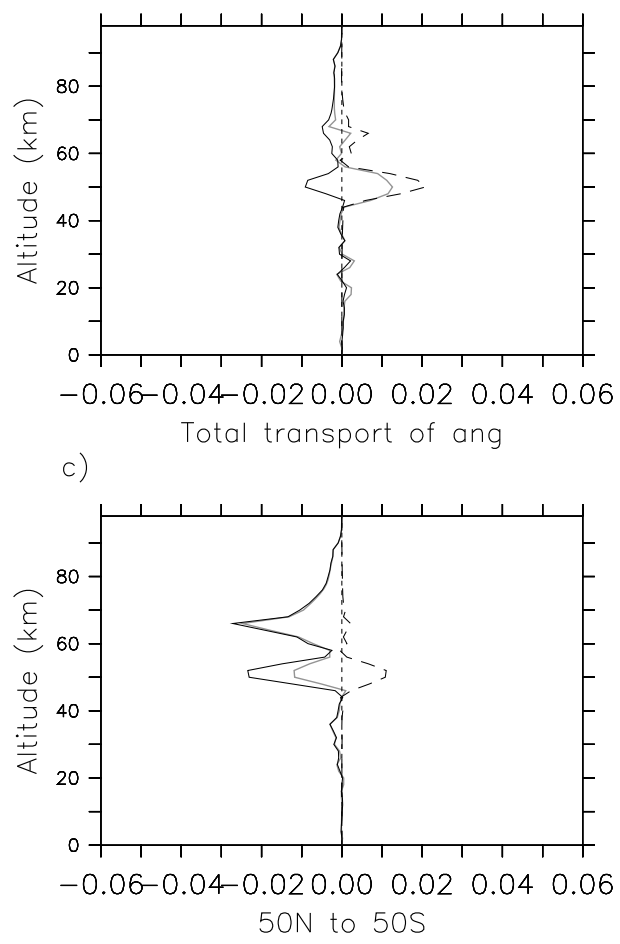

b)
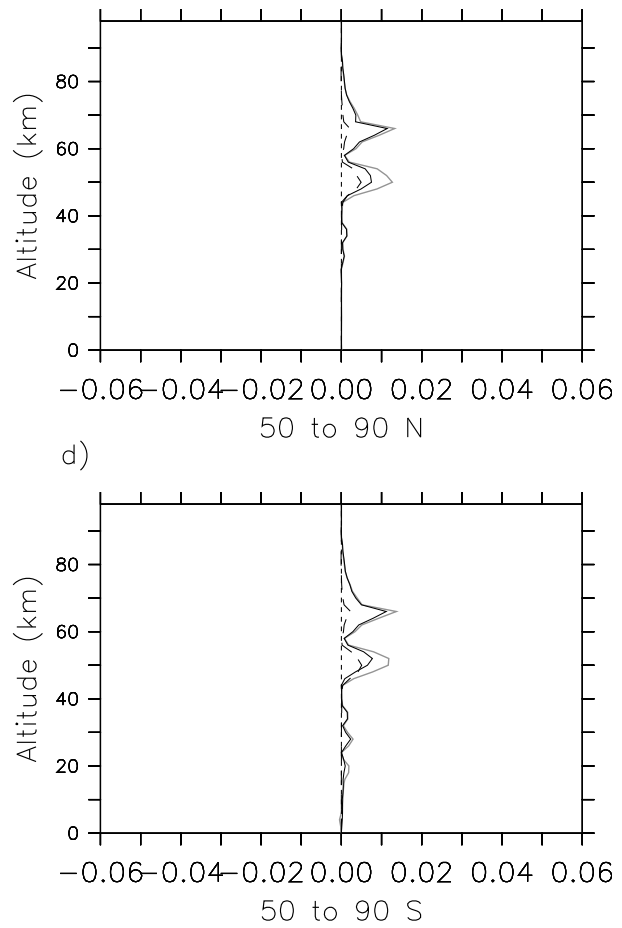

Figure 11. Vertical transport of angular momentum in the simulation without diurnal cycle. Black solid line, mean meridional circulation; dashed line, transients; dotted line, stationary waves; gray solid line, total. Temporal averages were done over days 300 to 304. (a) The relative angular momentum vertical transport is integrated for all latitudes at each given altitude and is divided by the latitudinal sum of the mass at this altitude. The relative angular momentum vertical transport is integrated only for a fraction of the latitudes ((b) $50^{\circ} \mathrm{N}-90^{\circ} \mathrm{N}$, (c) $50^{\circ} \mathrm{N}-50^{\circ} \mathrm{S}$, and (d) $50^{\circ} \mathrm{S}-90^{\circ} \mathrm{S}$ ) but is still divided by the total integrated mass at this altitude, so that Figures $11 \mathrm{~b}, 11 \mathrm{c}$, and $11 \mathrm{~d}$ add to give the corresponding plot in Figure 11a. It must be noted, though, that the total integrated mass at a given altitude is varying exponentially with the altitude. Also, the vertical wind being in $\mathrm{Pa} / \mathrm{s}$, upward transport is negative and downward transport is positive.

equatorial regions (where it is pumped from the surface). The transport of angular momentum resulting from the advection scheme is confined to the convective region (see Figure 8). The mixing due to convection induces a small downward transport of angular momentum, associated with the vertical gradient of zonal wind. For both simulations with or without diurnal cycle, the variations of angular momentum due to this transport are, most of the time, roughly one order of magnitude less than the angular momentum variations due to the meridional circulation, leaving this mixing a second-order process.

\subsection{Angular Momentum Transport: Role of Thermal Tides}

[77] The role of angular momentum transport by thermal tides in the maintenance of superrotation within the clouds in equatorial regions has been studied by Newman and Leovy [1992]. They used a semispectral primitive equation model that included the zonal mean flow, and wave numbers 1 and 2. These waves corresponded to diurnal and semidiurnal tides, though they could also be excited by instabilities. The background zonal wind speed was imposed as the initial condition (with a maximum of $75 \mathrm{~m} \mathrm{~s}^{-1}$ at equator). Diurnal and semidiurnal tides were transporting angular momentum from above the clouds into the cloud region, accelerating the equatorial flow at these altitudes, and smoothing the latitudinal variations of the wind speed between the midlatitude jet and the equator. Newman and Leovy [1992] also ran their model without any waves, and in this case their results appeared to be similar to our results, discussed in the previous section, with a strong barotropically unstable high-latitude jet. Though the background wind field remained unexplained, this paper illustrated the role of vertical transport of angular momentum by the thermal tides. This mechanism is in agreement with our results, as described below.

[78] Takagi and Matsuda [2007] also explored the role of thermal tides, using a GCM with prescribed solar heating and Newtonian cooling toward a zonal averaged temperature field. The zonally average component of the solar heating was suppressed. This strongly affects the angular momentum transport by mean meridional circulation, which was then almost eliminated. Their results emphasized the role of deceleration of the zonal flow close to the surface by semidiurnal tides, inducing momentum pumping through surface friction. During the spin-up phase of their simulations, this deceleration close to the ground was obtained through waves that were not thermal tides, but the resulting 

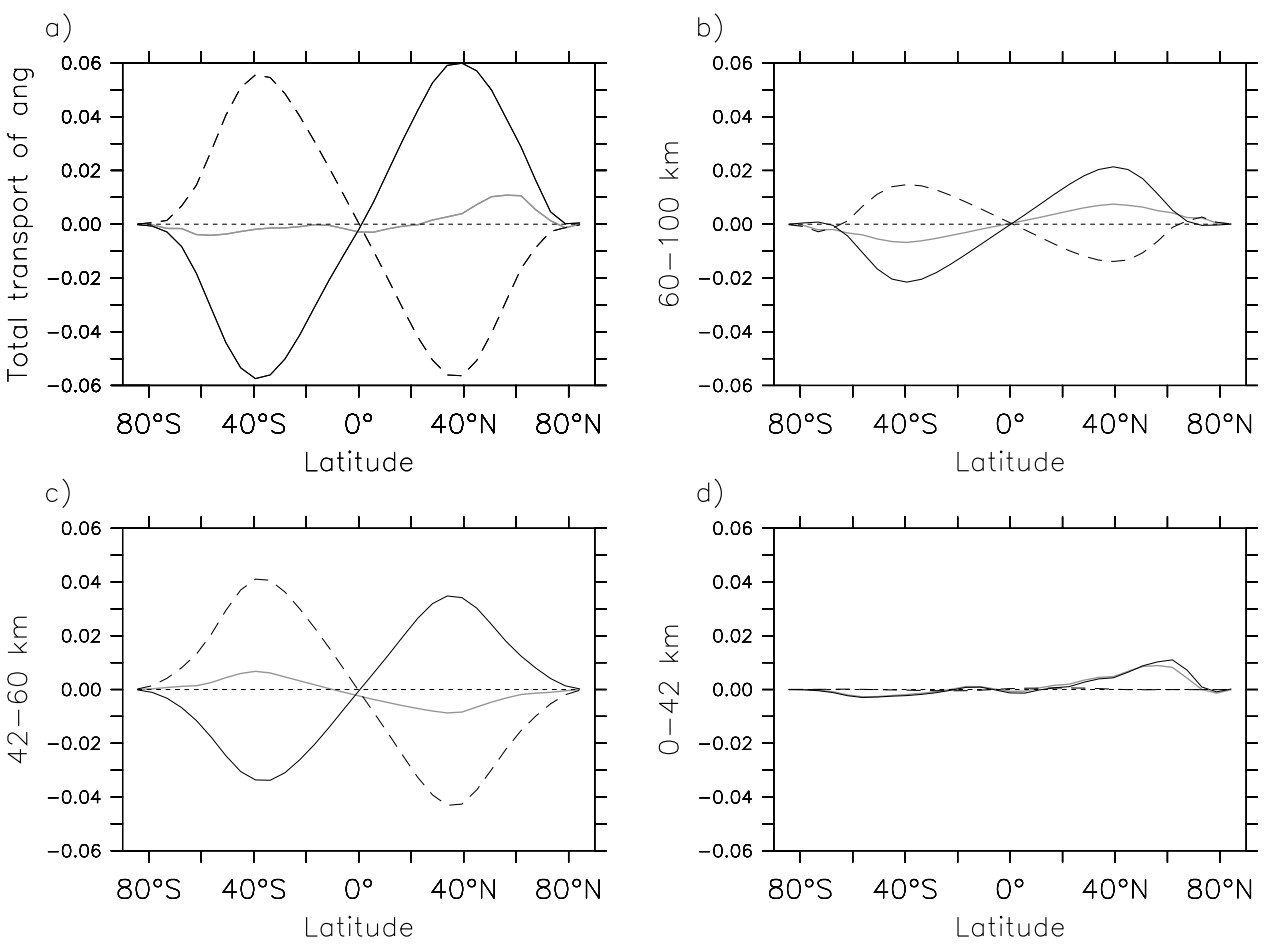

Figure 12. Latitudinal transport of angular momentum in the simulation without diurnal cycle (zonal average). Black solid line, mean meridional circulation; dashed line, transients; dotted line, stationary waves; gray solid line, total. Temporal averages were done over days 300 to 304 . (a) The relative angular momentum latitudinal transport is integrated for the entire column at each latitude and is divided by the total mass of the column. The relative angular momentum latitudinal transport is integrated only for a fraction of the column ((b) 60-100 km altitude, (c) 42-60 km, and (d) 0-42 km) but is still divided by the total mass of the entire column, so that Figures $12 \mathrm{~b}, 12 \mathrm{c}$, and $12 \mathrm{~d}$ add to give the corresponding plot in Figure 12a.

effect was similar, inducing pumping of angular momentum into the whole atmosphere. This effect was not obtained in our results.

[79] In our reference simulation where the thermal tides are present, the mechanism of angular momentum transport is different from the NDC case described in the previous section. As in the previous section, Figures 13 and 14 present the vertical and horizontal transport of angular momentum in this case, first globally integrated, then in different regions. The global vertical transport (Figure 13a) indicates stronger vertical exchanges than in the NDC case. The mean meridional circulation is able to transport angular momentum from the deep atmosphere up to regions above the clouds, despite the fact that the meridional stream function shows stacked cells. This may be explained by the fact that all these cells are dominantly direct. In the horizontal, the global plots (Figure 14a) are quite similar to the NDC case, though the exchanges are less intense. It is still compatible with the GRW mechanism, in the sense that the mean meridional circulation transports angular momentum poleward, while the transients bring it back toward the equator. However, no high-latitude jets are forming here, and therefore these transients may be different from the transients seen in the NDC case.

[80] Again, a region by region analysis helps us understand the angular momentum budget. In the vertical direc- tion, Figure 13c shows that the downward transport by transients (thermal tides) is very strong in the equatorial region, in the clouds (roughly between 45 and $80 \mathrm{~km}$ altitude, where the solar flux absorption is the strongest). In the polar regions, as in the NDC case, both the mean meridional circulation (dominant) and the transients transport angular momentum downward. For the mean meridional circulation, the ratio between the upward transport in the equatorial region and the downward transport in polar region is much higher than in the NDC simulation.

[81] This strong equatorial downward transport of angular momentum by the thermal tides in the cloud region is consistent with the work of Newman and Leovy [1992]. It helps maintaining the maximum of the zonal winds in this region, in the altitude range 45 to $80 \mathrm{~km}$. The peak of the zonal wind is located around $70 \mathrm{~km}$ altitude, consistent with observations, and it is lower than in the NDC case. The mean meridional circulation, though transporting angular momentum in a similar way as in the NDC case, is not strong enough here to induce a shift of the peak zonal wind speed toward the high-latitude jets.

[82] In our reference simulation, as was discussed in section 3, the latitudinal distribution of the zonal wind at cloud top is not as uniform in latitude as is observed in the Venus atmosphere. This suggests that the influence of the thermal tides is too large compared to the mean meridional 
a)
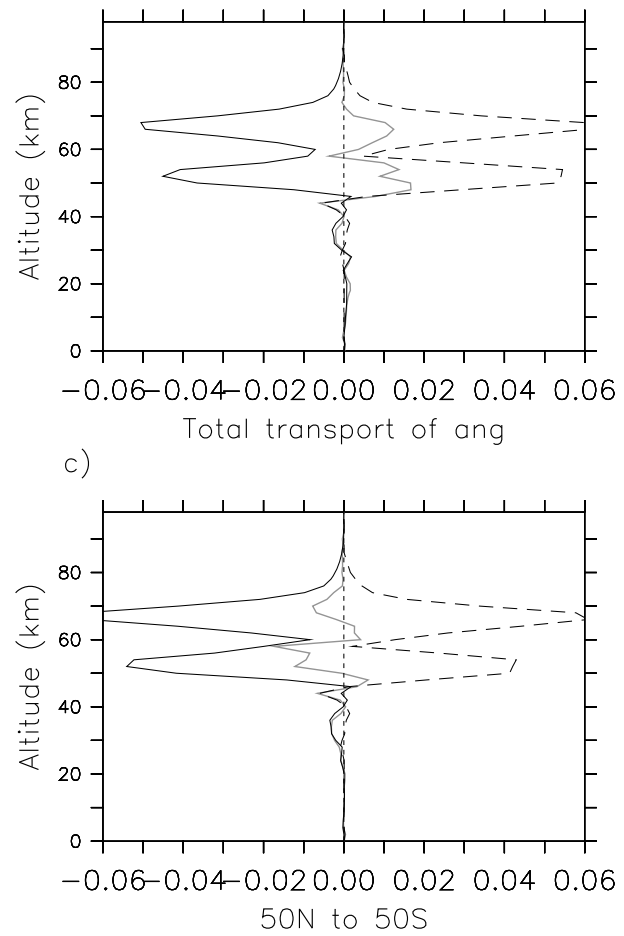

b)

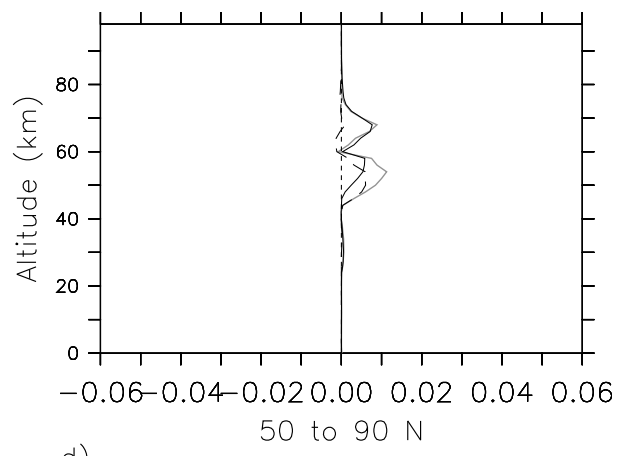

d)

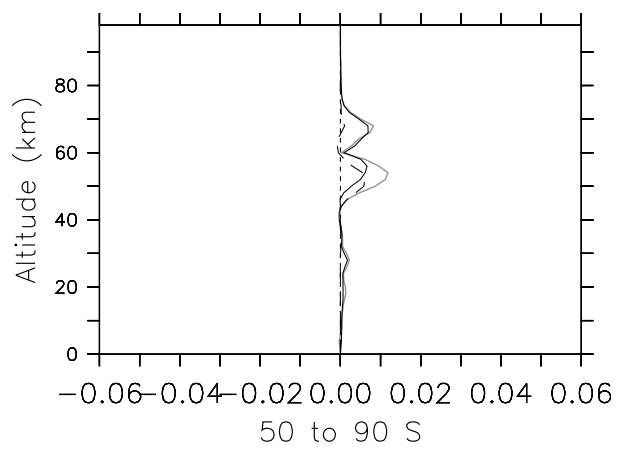

Figure 13. Same as Figure 11 but for the reference simulation with diurnal cycle. Temporal averages were done over days 350 to 354 .

latitudinal transport of angular momentum. This may be related to the misrepresentation of the latitudinal gradient of temperature (increasing this gradient would certainly increase the amplitude of the mean meridional circulation), or too weak global zonal wind below the clouds (which may be affecting both the global amplitude of the peak zonal wind and the global angular momentum transport budget).

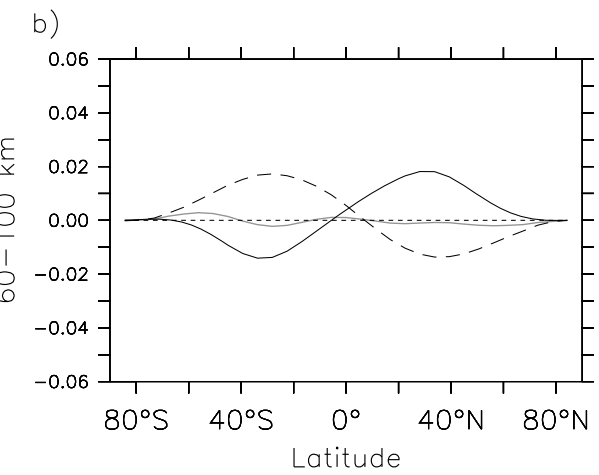

d)
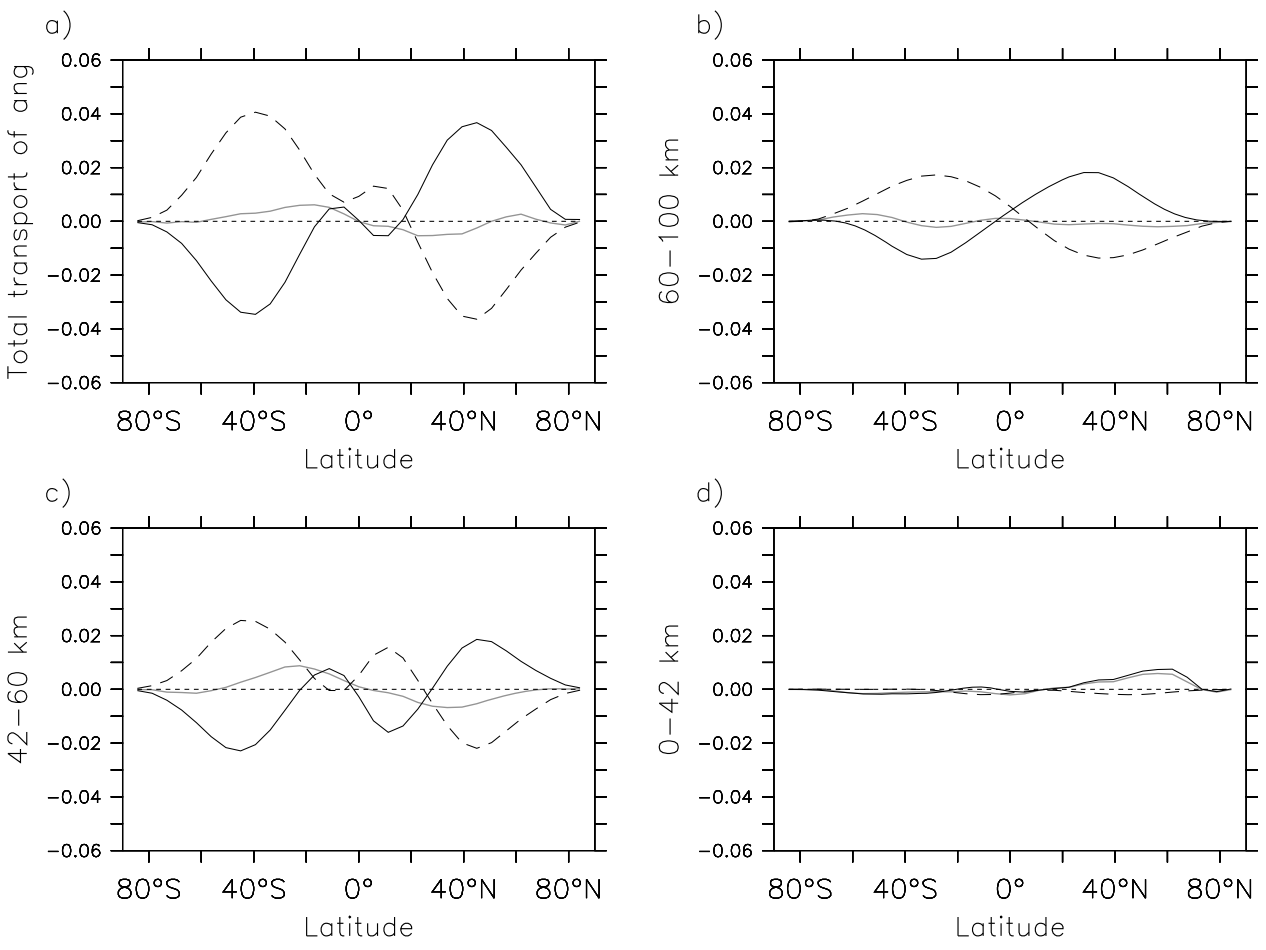

Figure 14. Same as Figure 12 but for the reference simulation with diurnal cycle. Temporal averages were done over days 350 to 354 . 
Reference
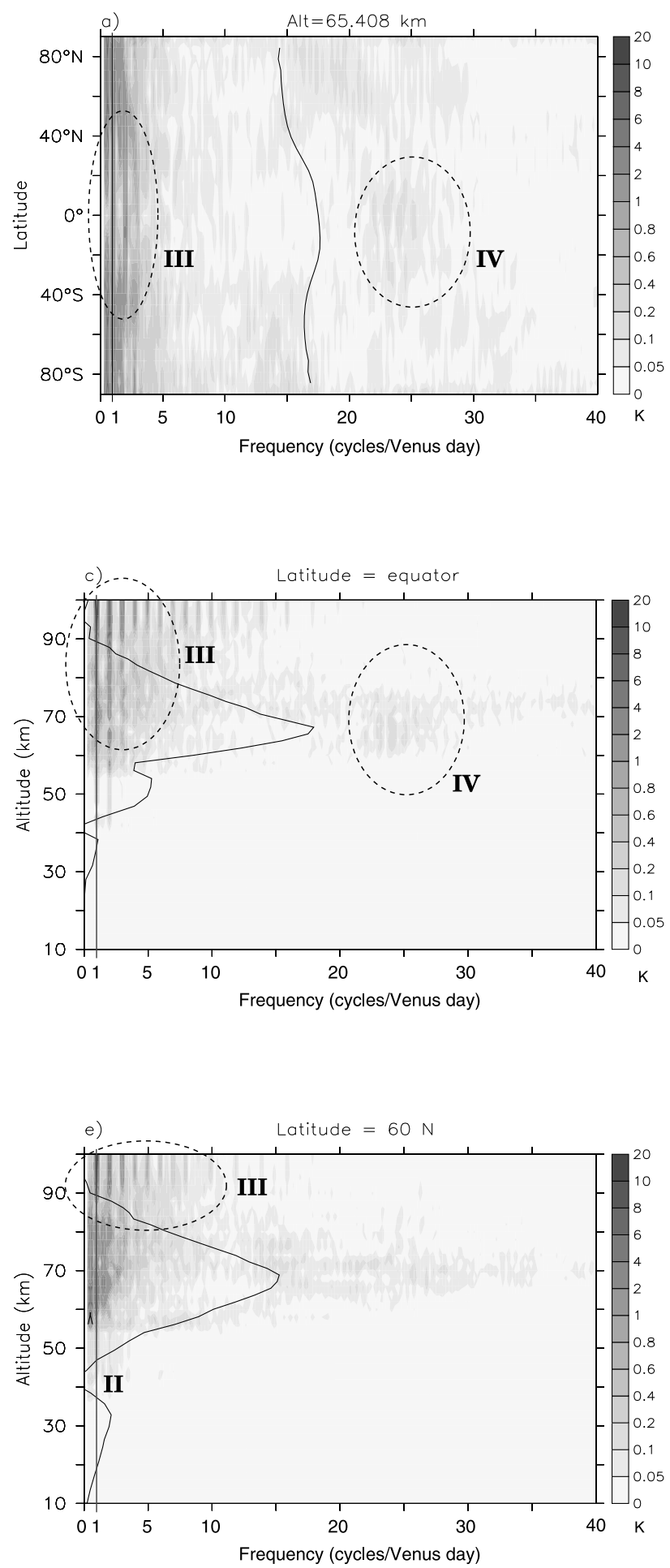

No diurnal cycle
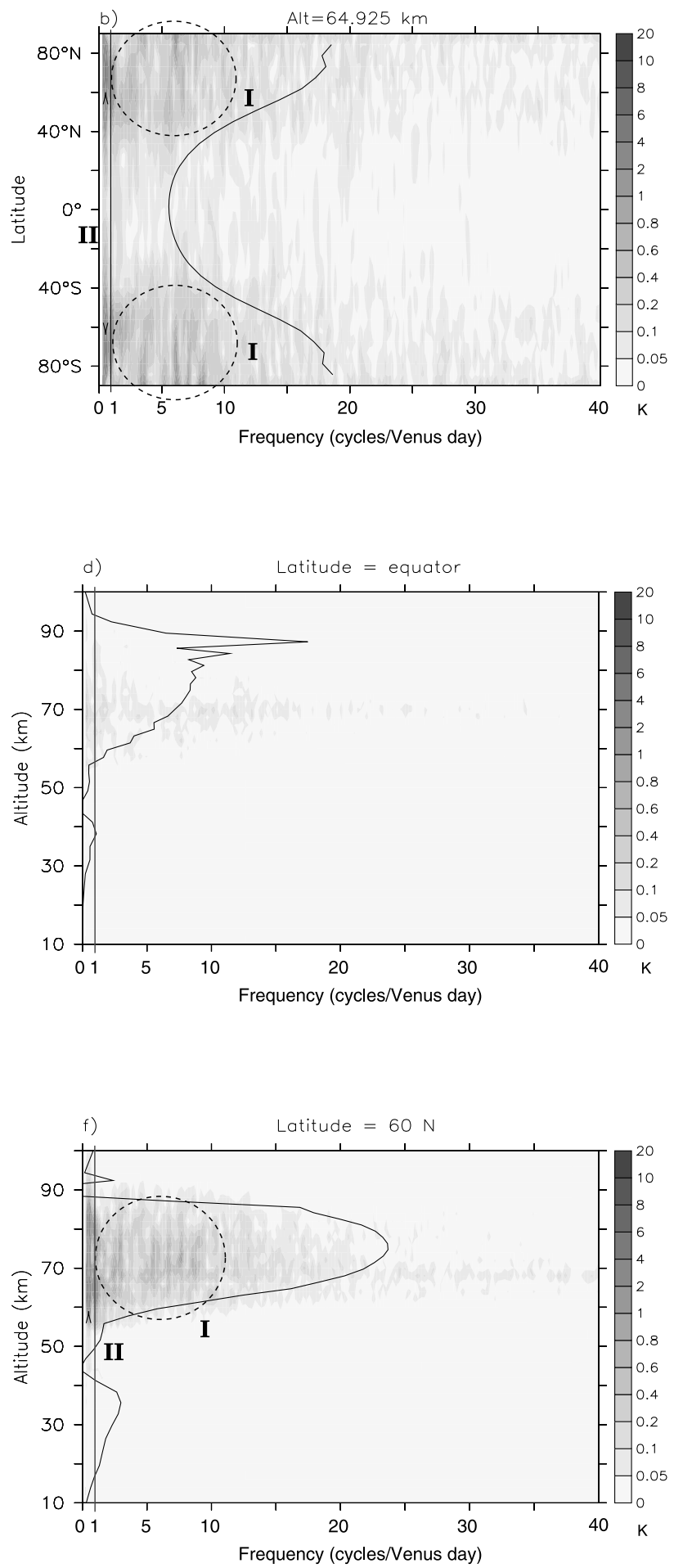

Figure 15. Amplitude frequency spectrum for the temperature at longitude $=0^{\circ}$ for $(\mathrm{a}, \mathrm{c}, \mathrm{e})$ the reference simulation (days 350-354) and (b, d, f) the NDC simulation (days 300-304). Figures 15a and 15b are around $65 \mathrm{~km}$, as a function of latitude; Figures $15 \mathrm{c}$ and $15 \mathrm{~d}$ are at the equator, as a function of altitude; and Figures $15 \mathrm{e}$ and $15 \mathrm{f}$ are at $60^{\circ} \mathrm{N}$, as a function of altitude. The solid curve is the mean zonal rotation speed of the flow (same unit as the frequency). A solid line also emphasizes the diurnal frequency. The different types of wave activity are indicated with dashed areas, bold arrows, and roman numbers (see text). 

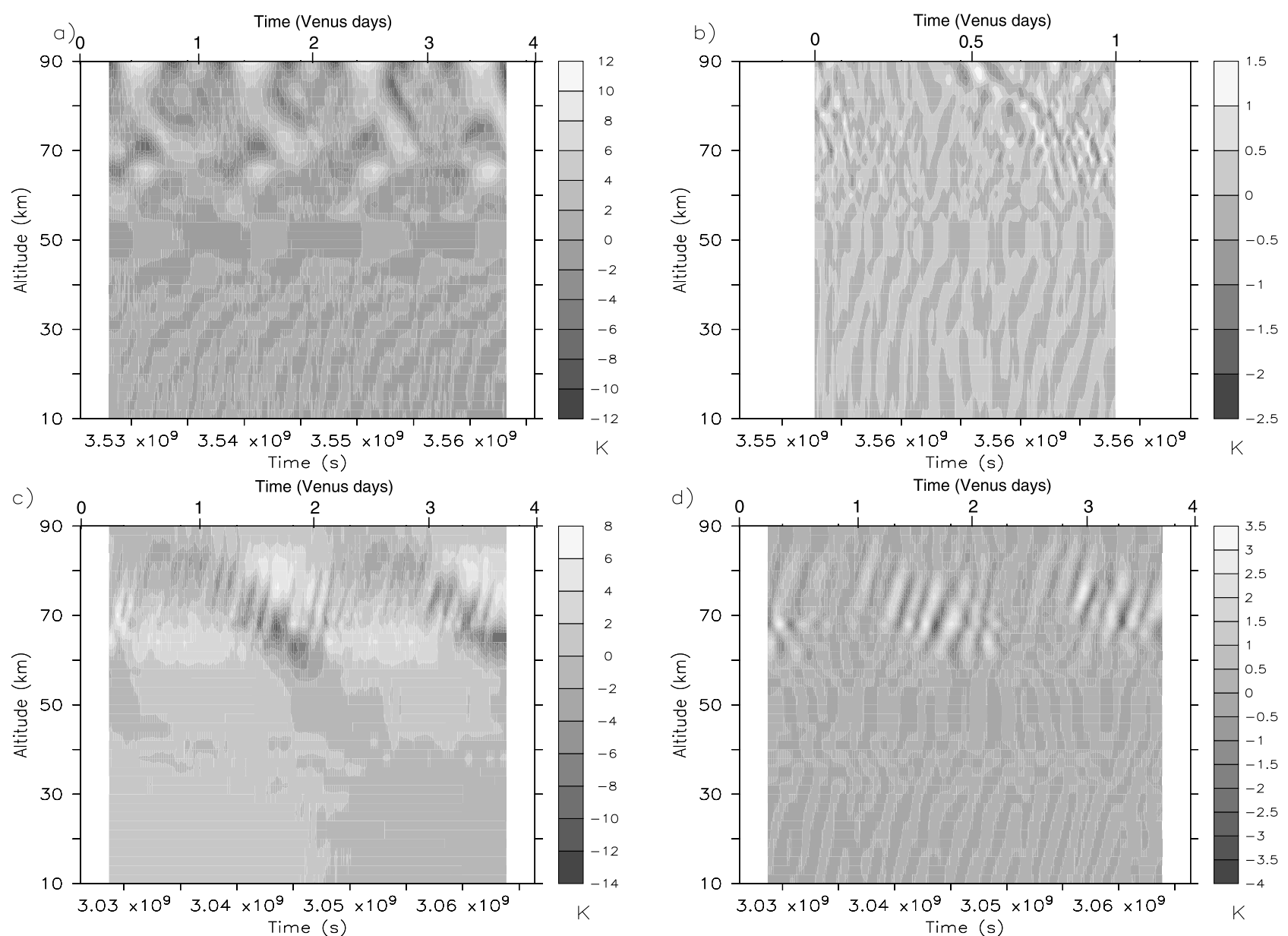

Figure 16. Altitude-time Hovmöller plots of temperature variability $\left(T^{\prime}=T-\bar{T}\right)$ at longitude $=0$. (a) At the equator, for the reference simulation, during four Venus days (days 350-354); the dominant signal is the diurnal tide. (b) Same as Figure 16a, but the signal is filtered for frequencies higher than $7 \times 10^{-7} \mathrm{~Hz}$ (periods around 16 Earth days) and only one Venus day is plotted, showing the type IV waves. (c) At $60^{\circ}$ latitude, for the NDC simulation, during four Venus days (days 300-304); the dominant signal is the type II quasi-bidiurnal wave. (d) Same as Figure 16c, but the signal is filtered for frequencies between roughly $3 \times 10^{-7}$ and $7 \times 10^{-7} \mathrm{~Hz}$ (periods between 16 to 38 Earth days), showing the type I waves.

[83] We have also performed the same analysis for the case without topography, to evaluate the influence of this parameter on the study. Figures (not shown) and analysis are then very similar to the case with topography presented in Figures 13 and 14.

\subsection{Wave Analysis}

[84] A Fourier transform analysis has been performed on the temperature field on high-frequency outputs from both the reference and the NDC simulations: 100 points per Venus day, during four days run after the initial simulations, days 350-354 for the reference run, and days 300-304 for the NDC simulation. Altitude-frequency plots at the equator and $60^{\circ} \mathrm{N}$, and latitude-frequency plots at $65 \mathrm{~km}$ altitude are presented in Figure 15.

[85] When the diurnal cycle is not taken into account (Figures 15b, 15d, and 15f), the dominant waves are seen in middle- to high-latitude regions, between 50 and $90 \mathrm{~km}$ altitude. We will refer to them as type I, for simplicity. Their amplitude is no larger than $1 \mathrm{~K}$. These waves have phase speeds that are slower than the zonal wind flow, i.e. they propagate in the opposite direction relative to the mean flow (as Rossby waves do).

[86] A very long period wave, quasi-bidiurnal, referred to as type II, is visible at high latitudes (above $50^{\circ}$ ), mainly within the upper cloud (60-70 $\mathrm{km}$ altitude), with an amplitude as high as $5 \mathrm{~K}$.

[87] As the zonal wind in the cloud region decreases with time in the NDC simulation, the amplitude of both types of waves is decreasing accordingly, when plotting them after 350 Venus days (not shown here).

[88] Taking into account the diurnal cycle induces large modifications to this picture. The dominant waves are now the diurnal tide and its harmonics, located at all latitudes, above $40 \mathrm{~km}$ altitude (type III). The amplitude of the tides is maximum at higher altitudes: within and above the clouds, it is around $5 \mathrm{~K}$ for the diurnal tide (but it is as high as $10 \mathrm{~K}$ or more close to the top of the model), and around $3 \mathrm{~K}$ for the semidiurnal tide. At high latitudes, the type I and II waves also seem to be present, as in the NDC simulation. Type II waves have even larger amplitudes, up to $10 \mathrm{~K}$. Another type of waves (type IV) is visible mainly in low latitude 
a)

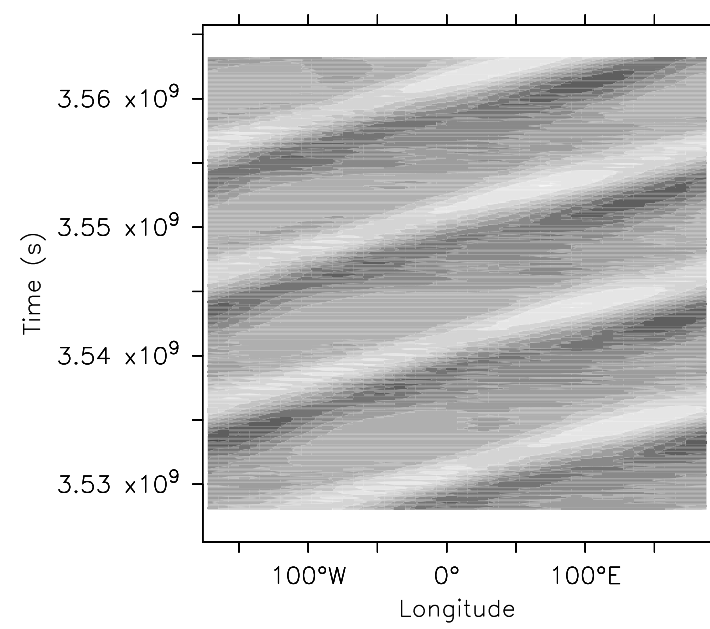

c)

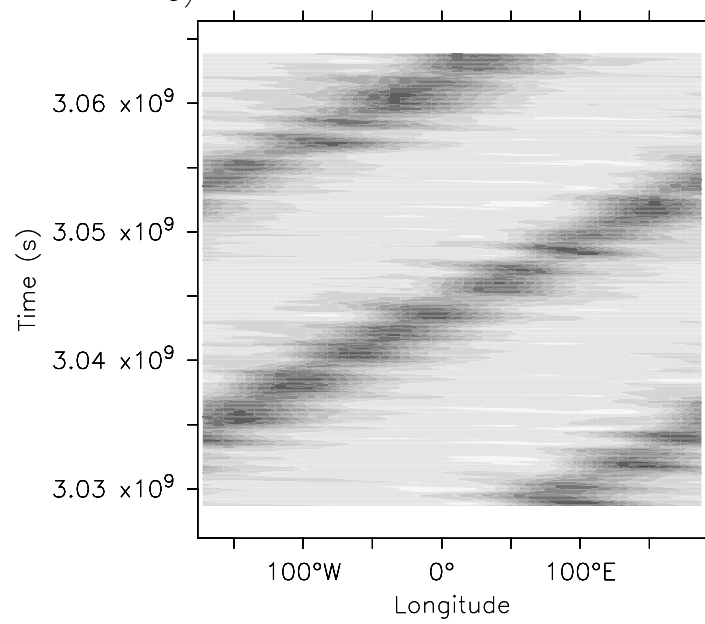

b)
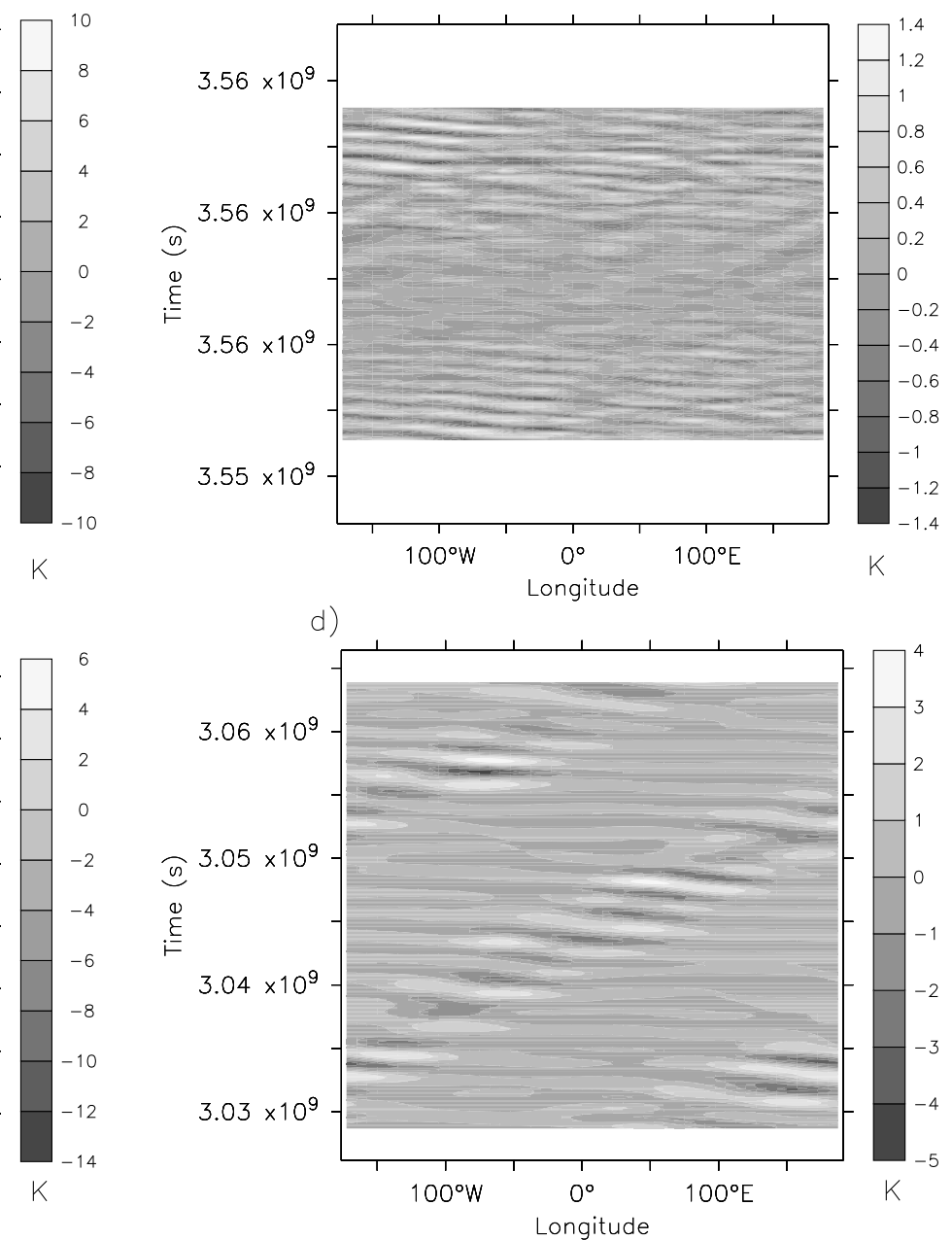

d)

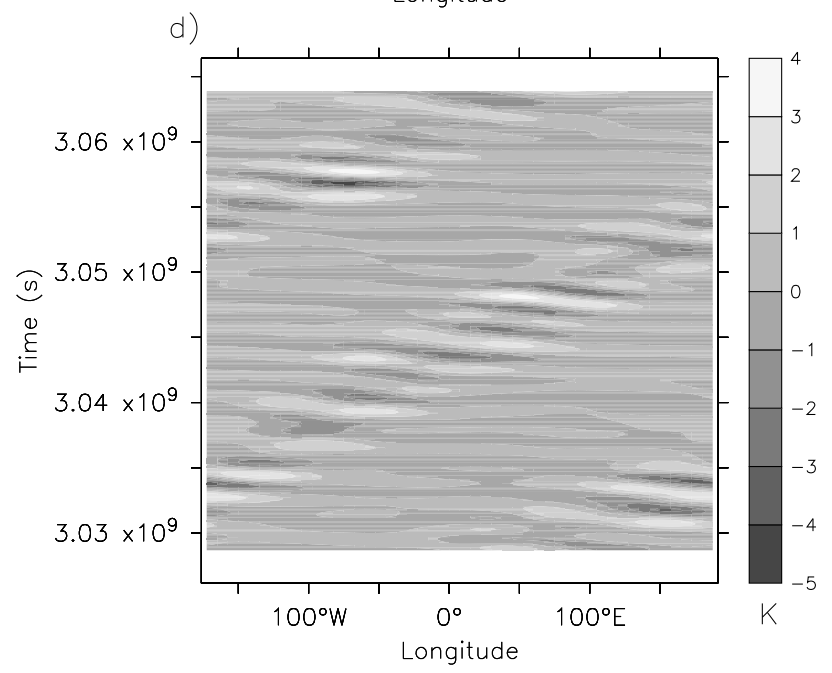

Figure 17. Longitude-time Hovmöller plots of temperature variability $(T=T-\bar{T})$ at $66 \mathrm{~km}$ altitude. (a) At the equator, for the reference simulation, during four Venus days (days 350-354); the dominant signal is the diurnal tide. (b) Same as Figure 17a, but the signal is filtered for frequencies higher than $7 \times 10^{-7} \mathrm{~Hz}$ (periods around 16 Earth days) and only one Venus day is plotted, showing the type IV waves. (c) At $60^{\circ}$ latitude, for the NDC simulation, during four Venus days (days 300-304); the dominant signal is the type II quasi-bidiurnal wave. (d) Same as Figure 17c, but the signal is filtered for frequencies between roughly $3 \times 10^{-7}$ and $7 \times 10^{-7} \mathrm{~Hz}$ (periods between 16 to 38 Earth days), showing the type I waves.

regions, between 60 and $70 \mathrm{~km}$ altitude (upper clouds), at high frequencies (around $2.5 \times 10^{-6} \mathrm{~Hz}$ ). The period of these waves is around 4 to 5 Earth days, so there may be a relation with the wave structures observed at the cloud top at ultraviolet wavelengths [Del Genio and Rossow, 1990]. However, their amplitude is very small, of order $0.1 \mathrm{~K}$. It must be kept in mind that the modeled zonal wind in this simulation is around half of the observed value, which changes the relative wave speed of these waves compared to observed ones.

[89] The location of type I and II waves appear to be strongly correlated with regions of strong zonal winds, while type III waves are not. This is consistent with type I and II waves being generated by instabilities associated with zonal jets, while diurnal tides have no connection to zonal winds. For type IV waves, a connection to equatorial zonal winds is also possible.
[90] The convective region between $47 \mathrm{~km}$ altitude (cloud base) and roughly $55 \mathrm{~km}$ altitude is clearly visible in both simulations, as it inhibits the wave activity.

[91] Figures 16 and 17 illustrate these dominant waves, using two-dimensional plots with time as one dimension (also known as Hovmöller plots): altitude vs time, then longitude vs time at $66 \mathrm{~km}$ altitude. Figures 16 and 17 display the temperature temporal variability $T=T-\bar{T}$, i.e. the difference between the temperature field and its average over four Venus days. Figures 16a and 17a shows equatorial temperature variations in the reference simulation, which emphasizes the thermal tides (type III). Then in Figures 16b and $17 \mathrm{~b}$, the same temperature variations are filtered (the cutoff frequency is around $7 \times 10^{-7} \mathrm{~Hz}$, which corresponds to a period around 16 Earth days) to show the high-frequency type IV waves. In Figures 16c, 16d, 17c, and 17d, temperature variations in the NDC simulation are shown at $60^{\circ} \mathrm{N}$ : first, the total signal shows the type II, long period waves, then the 
signal is filtered, keeping frequencies between $3 \times 10^{-7}$ and $7 \times 10^{-7} \mathrm{~Hz}$ (periods between 16 and 38 Earth days) and therefore type I waves. Types II and III waves propagate eastward, in the same direction as the diurnal forcing. Types I and IV propagate westward, along with the zonal flow.

[92] Based on an analysis of filtered $\left[\overline{v^{\prime} u^{\prime}}\right]$ (the transport term for zonal wind, due to transients), using cutoff frequencies around $3 \times 10^{-7} \mathrm{~Hz}$ (3 (Venus days) ${ }^{-1}$, roughly 38 Earth days) and $7 \times 10^{-7} \mathrm{~Hz}$ (7 (Venus days) $)^{-1}$, roughly 16 Earth days), it appears that both in the reference and NDC simulations, the transport of angular momentum by waves is essentially done through long-period waves (over 38 Earth days). These waves may be type III (thermal tides) for the reference simulation, and types I (longest-period part of them) and II. The origin and role of the type II, quasi-bidiurnal waves modeled at high latitudes is not clear. However, the variations observed in the cloud structure at high latitudes, which are not taken into account in this GCM, may affect the flow through modified radiative transfer, and therefore affect these waves. This should be tested when a more realistic latitudinal structure for the clouds is used in the radiative transfer computations.

[93] As in section 4.2, the possible role of topography has been evaluated by the same analysis on the simulation without topography. Figures (not shown) and analysis are again very similar to the case with topography presented in this section.

\section{Conclusion}

[94] Based on the LMDZ general circulation model used for the Earth, we have developed a general circulation model for the Venus atmosphere, from the surface up to roughly $100 \mathrm{~km}$ altitude. This new GCM takes into account the topography, the diurnal cycle, the dependence of the specific heat to the temperature, and a consistent radiative transfer module. The temperature dependence of $C_{p}$ is very large in the Venus atmosphere, and this relation induced modifications in the definition of the potential temperature that is used as a basic variable in the dynamical core. Though this temperature dependence of $C_{p}$ affects the temperature structure of the atmosphere, the impact over the wind structure appears to be of second order, as shown in this work.

[95] The radiative transfer developed for infrared based on net exchange rates matrices [Eymet et al., 2009] allows a consistent computation of the temperature field, which is different from previous GCMs of Venus' atmosphere, which were based on simplified forcing of the temperature structure. To illustrate the need for such a realistic computation of temperature, we have also run our GCM with simplified temperature forcing. These simulations show results fairly similar to previously published works. Comparison between both runs shows how the zonal and meridional wind fields are affected by such a simplification.

[96] After a long spin-up phase, covering at least 150 Venus days (or more than 50 Earth years), we analyzed the modeled zonal and meridional circulations. Superrotation is obtained at the cloud level, above roughly $40 \mathrm{~km}$ altitude, but below this altitude, the zonal wind does not increase to the observed values. This discrepancy is a major pending question. The sensitivity of the simulations to the choices of boundary layer scheme and parameters is currently under investigation, as preliminary tests indicate that the deep atmospheric circulation may be significantly affected by these choices. The possible role of gravity waves generated close to the surface need also to be assessed by further studies. The meridional circulation consists of equator-topole cells, with the dominant one located within the cloud layers. The modeled temperature structure is globally quite consistent with observations, though several discrepancies need to be explained and improved: the atmosphere is too stable in the lowest $15 \mathrm{~km}$, and the latitudinal contrast between equator and high latitudes in the upper cloud (the "cold collar") is not as large as observed. This may be related to the fact that clouds are considered uniform with altitude for the moment. This probable coupling between cloud structure, radiation field and circulation needs to be taken into account in future improvements of the GCM. It may also be possible that this discrepancy is of dynamical origin, due to the structure of the zonal wind field in the cloud region. A convective layer is found between the base of the clouds (around $47 \mathrm{~km}$ ), heated from the deep atmosphere below and the middle of the clouds $(55-60 \mathrm{~km}$ altitude), region that is able to cool directly to space; this is consistent with observation of the stability structure above $40 \mathrm{~km}$.

[97] Conservation of angular momentum is tested, and though it is not perfect in the model, superrotation cannot be produced artificially from the residual advection errors. The transport of angular momentum has been analyzed, and comparison between the reference simulation and a sensitivity simulation performed without a diurnal cycle clearly illustrates the role played by the thermal tides in the equatorial region.

[98] When the diurnal cycle is shut down, the transport of angular momentum is consistent with the Gierasch-RossowWilliams mechanism, where horizontal transport of angular momentum by waves at midlatitudes within the cloud layers compensates the poleward transport of angular momentum by the mean meridional circulation. The resulting zonal wind field displays high-latitude jets above the clouds, and instabilities are able to develop on the equatorial flank of these jets. However, this structure is not stable in this test, and the jets fade away after 100 Venus days.

[99] The mechanism controlling equatorial superrotation in the reference case where thermal tides are present is more complex than suggested by the GRW mechanism. The mean meridional circulation transports angular momentum upward (despite layered Hadley cells), and poleward, but this poleward transport is weakened by the thermal tides. These tides add a significant downward transport of angular momentum in the equatorial region, which allows accumulation of angular momentum at low latitudes, forbidding the build of the high-latitude jets. However, horizontal equatorward transport of angular momentum by waves is still obtained in the cloud layer to compensate the mean meridional circulation horizontal transport. The resulting zonal wind field displays a stable maximum at the equator. The observed zonal wind profile is intermediate between these two situations, with roughly constant zonal winds equatorward of $50^{\circ}$ latitude. This discrepancy in the balance between downward transport of angular momentum by thermal tides and horizontal transport by the mean meridi- 
onal circulation (and transient waves) in the cloud region may be related to the misrepresentation of the latitudinal temperature gradients in the clouds, or to the absence of superrotation below the clouds.

[100] An analysis of the waves present in the reference simulation and in the simulation without diurnal cycle has been done, using Fourier transform of the temperature field. When no diurnal cycle is taken into account, waves are present at high latitudes, in the region of maximum winds. A second type of waves, of very long period (quasi-bidiurnal), are also present. With the diurnal cycle, thermal tides are clearly identified. As mentioned above, their role in the vertical transport of angular momentum is crucial. Within the upper cloud, short period waves (4 to 5 Earth day period) are also seen in the equatorial region.

[101] Due to the discrepancy between modeled and observed zonal wind fields, the superrotation phenomenon is not yet fully understood. However, the role of thermal tides is emphasized, as well as the importance to compute accurately the radiative transfer and the temperature field. The impact of latitudinal variations in the cloud structure (associated with coupling between clouds, temperature structure and dynamics) need to be fully investigated in future developments of the model.

[102] Acknowledgments. This work has been supported by the Centre National d'Etudes Spatiales, by the project Exoclimats financed by the Agence Nationale de la Recherche, and by the computation facilities of the Institut du Développement et des Ressources en Informatique Scientifique.

\section{References}

Crisp, D. (1986), Radiative forcing of the Venus mesosphere. I-Solar fluxes and heating rates, Icarus, 67, 484-514.

Crisp, D., and D. Titov (1997), The thermal balance of the Venus atmosphere, in Venus II: Geology, Geophysics, Atmosphere, and Solar Wind Environment, edited by S. W. Bougher, D. M. Hunten, and R. J. Phillips, pp. 353-384, Univ. of Ariz. Press, Tucson.

Del Genio, A. D., and W. B. Rossow (1990), Planetary-scale waves and the cyclic nature of cloud top dynamics on Venus, J. Atmos. Sci., 47, 293 318.

Del Genio, A. D., and W. Zhou (1996), Simulations of superrotation on slowly rotating planets: Sensitivity to rotation and initial conditions, Icarus, 120, 332-343.

Del Genio, A. D., W. Zhou, and T. P. Eichler (1993), Equatorial superrotation in a slowly rotating GCM: Implications for Titan and Venus, Icarus, $101,1-17$

Dufresne, J.-L., R. Fournier, C. Hourdin, and F. Hourdin (2005), Net exchange reformulation of radiative transfer in the $\mathrm{CO}_{2} 15 \mu \mathrm{m}$ band on Mars, J. Atmos. Sci., 62, 3303-3319, doi:10.1175/JAS3537.1.

Eymet, V., J.-L. Dufresne, R. Fournier, and S. Blanco (2005), A boundary based net exchange Monte-Carlo method for absorbing and scattering thick medium, J. Quant. Spectrosc. Radiat. Transfer, 95, 27-46.

Eymet, V., R. Fournier, J.-L. Dufresne, S. Lebonnois, F. Hourdin, and M. A. Bullock (2009), Net exchange parameterization of thermal infrared radiative transfer in Venus' atmosphere, J. Geophys. Res., 114, E11008, doi:10.1029/2008JE003276.

Fels, S. B. (1986), An approximate analytical method for calculating tides in the atmosphere of Venus, J. Atmos. Sci., 43, 2757-2772.

Ford, P. G., and G. H. Pettengill (1992), Venus topography and kilometerscale slopes, J. Geophys. Res., 97, 13,103-13,114.

Forget, F., F. Hourdin, R. Fournier, C. Hourdin, O. Talagrand, M. Collins, S. R. Lewis, P. L. Read, and J.-P. Huot (1999), Improved general circulation models of the Martian atmosphere from the surface to above 80 km, J. Geophys. Res., 104, 24,155-24,176.

Gierasch, P. (1975), Meridional circulation and the maintenance of the Venus atmospheric rotation, J. Atmos. Sci., 32, 1038-1044.

Gierasch, P. J., et al. (1997), The general circulation of the Venus atmosphere: An assessment, in Venus II: Geology, Geophysics, Atmosphere, and Solar Wind Environment, edited by S. W. Bougher, D. M. Hunten, and R. J. Phillips, pp. 459-500, Univ. of Ariz. Press, Tucson.
Grassi, D., P. Drossart, G. Piccioni, N. I. Ignatiev, L. V. Zasova, A. Adriani, M. L. Moriconi, P. G. J. Irwin, A. Negrão, and A. Migliorini (2008), Retrieval of air temperature profiles in the Venusian mesosphere from VIRTIS-M data: Description and validation of algorithms, J. Geophys. Res., 113, E00B09, doi:10.1029/2008JE003075.

Herrnstein, A., and T. E. Dowling (2007), Effect of topography on the spinup of a Venus atmospheric model, J. Geophys. Res., 112, E04S08, doi:10.1029/2006JE002804

Hollingsworth, J. L., R. E. Young, G. Schubert, C. Covey, and A. S. Grossman (2007), A simple-physics global circulation model for Venus: Sensitivity assessments of atmospheric superrotation, Geophys. Res. Lett., 34, L05202, doi:10.1029/2006GL028567.

Hou, A. Y., S. B. Fels, and R. M. Goody (1990), Zonal superrotation above Venus' cloud base induced by the semidiurnal tide and the mean meridional circulation, J. Atmos. Sci., 47, 1894-1901.

Hourdin, F., P. Le Van, O. Talagrand, R. Courtin, D. Gautier, and C. P. McKay (1992), Numerical simulation of the circulation of the atmosphere of Titan, in Symposium on Titan: Proceedings, Toulouse, France, 9-12 September 1991, edited by B. Kaldeich, Eur. Space Agency Spec. Publ., ESA SP338, 101-106.

Hourdin, F., P. Le Van, F. Forget, and O. Talagrand (1993), Meteorological variability and the annual surface pressure cycle on Mars, J. Atmos. Sci., $50,3625-3640$

Hourdin, F., O. Talagrand, R. Sadourny, R. Courtin, D. Gautier, and C. P. McKay (1995), Numerical simulation of the general circulation of the atmosphere of Titan, Icarus, 117, 358-374.

Hourdin, F., S. Lebonnois, D. Luz, and P. Rannou (2004), Titan's stratospheric composition driven by condensation and dynamics, J. Geophys. Res., 109, E12005, doi:10.1029/2004JE002282.

Hourdin, F., et al. (2006), The LMDZ4 general circulation model: Climate performance and sensitivity to parameterized physics with emphasis on tropical convection, Clim. Dyn., 27, 787-813, doi:10.1007/s00382-0060158-0.

Ikeda, K., M. Yamamoto, and M. Takahashi (2007), Superrotation of the Venus atmosphere simulated by an atmospheric general circulation model, in Proceedings of IUGG XXIV General Assembly, edited by L. Ubertini et al., p. 488, Umbria Sci. Meet. Assoc., Perugia, Italy.

Karatekin, O., V. Dehant, and S. Lebonnois (2009), Length of day variations of Venus, Bull. Am. Astron. Soc., 41, 561 .

Lebonnois, S., F. Hourdin, V. Eymet, R. Fournier, and J.-L. Dufresne (2005), A new Venus general circulation model, in the context of the Venus-Express mission, Bull. Am. Astron. Soc., 37, 742.

Lee, C. (2006), Modelling of the atmosphere of Venus, Ph.D. thesis, Univ. of Oxford, Oxford, U. K.

Lee, C., S. R. Lewis, and P. L. Read (2005), A numerical model of the atmosphere of Venus, Adv. Space Res., 36, 2142-2145.

Lee, C., S. R. Lewis, and P. L. Read (2007), Superrotation in a Venus general circulation model, J. Geophys. Res., 112, E04S11, doi:10.1029 2006JE002874

Leovy, C. B., and N. L. Baker (1987), Zonal winds near Venus' cloud top level: A model study of the interaction between the zonal mean circulation and the semidiurnal tide, Icarus, 69, 202-220.

Limaye, S. S. (2007), Venus atmospheric circulation: Known and unknown, J. Geophys. Res., 112, E04S09, doi:10.1029/2006JE002814.

Lott, F., and M. Miller (1997), A new sub-grid scale orographic drag parameterization: Its formulation and testing, Q.J. R. Meteorol. Soc., $123,101-128$

Louis, J. (1979), A parametric model of vertical eddy fluxes in the atmosphere, Boundary Layer Meteorol., 17, 187-202.

Luz, D., and F. Hourdin (2003), Latitudinal transport by barotropic waves in Titan's stratosphere. I. General properties from a horizontal shallowwater model, Icarus, 166, 328-342.

Newman, M., and C. B. Leovy (1992), Maintenance of strong rotational winds in Venus' middle atmosphere by thermal tides, Science, 257, $647-650$.

Pechman, J. B., and A. P. Ingersoll (1984), Thermal tides in the atmosphere of Venus: Comparison of model results with observations, J. Atmos. Sci., $41,3290-3313$.

Peralta, J., R. Hueso, and A. Sanchez-Lavega (2007), A reanalysis of Venus winds at two cloud levels from Galileo SSI images, Icarus, 190, 469-477.

Rannou, P., F. Hourdin, and C. P. McKay (2002), A wind origin for Titan's haze structure, Nature, 418, 853-856.

Rannou, P., F. Hourdin, C. P. McKay, and D. Luz (2004), A coupled dynamics-microphysics model of Titan's atmosphere, Icarus, 170, 443-462.

Rossow, W. B., and G. P. Williams (1979), Large-scale motion in the Venus stratosphere, J. Atmos. Sci., 36, 377-389. 
Rossow, W. B., S. B. Fels, and P. H. Stone (1980), Comments on "A threedimensional model of dynamical processes in the Venus atmosphere," J. Atmos. Sci., 37, 250-252.

Sánchez-Lavega, A., et al. (2008), Variable winds on Venus mapped in three dimensions, Geophys. Res. Lett., 35, L13204, doi:10.1029/ 2008 GL033817.

Schubert, G. (1983), General circulation and the dynamical state of the Venus atmosphere, in Venus, edited by D. M. Hunten et al., pp. 681765, Univ. of Ariz. Press, Tucson.

Schubert, G., and J. A. Whitehead (1969), The moving flame experiment with liquid mercury: Possible implications for the Venus atmosphere Science, 163, 71-72.

Seiff, A. (1983), Thermal structure of the atmosphere of Venus, in Venus, edited by D. M. Hunten et al., pp. 215-279, Univ. of Ariz. Press, Tucson.

Seiff, A., D. B. Kirk, R. E. Young, R. C. Blanchard, J. T. Findlay, G. M. Kelly, and S. C. Sommer (1980), Measurements of thermal structure and thermal contrasts in the atmosphere of Venus and related dynamical observations: Results from the four Pioneer Venus probes, J. Geophys. Res., 85, 7903-7933.

Seiff, A., J. T. Schofield, A. J. Kliore, F. W. Taylor, S. S. Limaye, H. E. Revercomb, L. A. Sromovsky, V. V. Kerzhanovich, V. I. Moroz, and M. Y. Marov (1985), Model of the structure of the atmosphere of Venus from surface to 100 kilometers altitude, Adv. Space Res., 5, 3-58.

Sromovsky, L. A., H. E. Revercomb, and V. E. Suomi (1985), Temperature structure in the lower atmosphere of Venus: New results derived from Pioneer Venus entry probe measurements, Icarus, 62, 458-493.

Takagi, M., and Y. Matsuda (2007), Effects of thermal tides on the Venus atmospheric superrotation, J. Geophys. Res., 112, D09112, doi:10.1029/ 2006JD007901.

Tellmann, S., M. Pätzold, B. Häusler, M. K. Bird, and G. L. Tyler (2009), Structure of the Venus neutral atmosphere as observed by the Radio Science experiment VeRa on Venus Express, J. Geophys. Res., 114, E00B36, doi:10.1029/2008JE003204.

Titov, D. V., F. W. Taylor, N. I. Svedhem, H. Ignatiev, W. J. Markiewicz, G. Piccioni, and P. Drossart (2008), Atmospheric structure and dynamics as the cause of ultraviolet markings in the clouds of Venus, Nature, 456 , 620-623, doi:10.1038/nature07466.

Tomasko, M. G., L. R. Doose, P. H. Smith, and A. P. Odell (1980), Measurements of the flux of sunlight in the atmosphere of Venus, J. Geophys. Res., 85, 8167-8186.

Wilson, C. F., S. Guerlet, P. G. J. Irwin, C. C. C. Tsang, F. W. Taylor, R. W. Carlson, P. Drossart, and G. Piccioni (2008), Evidence for anomalous cloud particles at the poles of Venus, J. Geophys. Res., 113, E00B13, doi:10.1029/2008JE003108.

Yamamoto, M., and M. Takahashi (2003), The fully developed superrotation simulated by a general circulation model of a Venus-like atmosphere, J. Atmos. Sci., 60, 561-574.

Yamamoto, M., and M. Takahashi (2004), Dynamics of Venus' superrotation: The eddy momentum transport processes newly found in a GCM, Geophys. Res. Lett., 31, L09701, doi:10.1029/2004GL019518.

Yamamoto, M., and M. Takahashi (2006), Superrotation maintained by meridional circulation and waves in a Venus-like AGCM, J. Atmos Sci., 63, 3296-3314.

Young, R. E., and J. B. Pollack (1977), A three-dimensional model of dynamical processes in the Venus atmosphere, J. Atmos. Sci., 34 , 1315-1351.

Young, R. E., and J. B. Pollack (1980), Reply, J. Atmos. Sci., 37, 253-255.

Zasova, L. V., N. I. Ignatiev, I. A. Khatuntsev, and V. Linkin (2007), Structure of the Venus atmosphere, Planet. Space Sci., 55, 1712-1728, doi:10.1016/j.pss.2007.01.011.

Zimbelman, J. R. (1986), The role of porosity in thermal inertia variations on basaltic lavas, Icarus, 68, 366-369.

A. Crespin, F. Forget, F. Hourdin, and S. Lebonnois, Laboratoire de Météorologie Dynamique, UMR 8539, IPSL, UPMC, CNRS, 4 place Jussieu, Box 99, F-75252 Paris CEDEX 05, France. (sebastien. lebonnois@lmd.jussieu.fr)

V. Eymet and R. Fournier, Laboratoire Plasma et Conversion de l'Energie, UMR 5213, UPS, CNRS, F-31071 Toulouse, France. 